\title{
Free-radical cyclisations of 2-aminoalka-2,5-dienenitriles
}

\author{
Chau-Chen Yang and Jim-Min Fang* \\ Department of Chemistry, National Taiwan University, Taipei, Taiwan 106, Republic of China
}

The intramolecular free-radical cyclisations of a series of 2-( $N$-methylanilino)alka-2,5-dienenitriles 5-16 have been studied. These compounds have two alkenyl groups orientated in equal proximity to the radical centres. One alkenyl group contains both the cyano and the $N$-methylanilino substituents, while the other contains either an electron-withdrawing or an electron-donating group. Intramolecular free-radical cyclisations occur exclusively or predominantly on the amino-cyano substituted alkenyl group (C-3 attack). The radical cyclisation of 4-(2-bromobenzyl)methyl-3,5-dimethyl-2-( $N$-methylanilino)hepta-2,5-diene-1,7dinitrile also occur exclusively at C-3.

We have demonstrated earlier that various cycloalkyl $\alpha$-amino nitriles 2 can be obtained in a stereoselective manner by the intramolecular free-radical cyclisations of $\alpha$-anilinoalkenenitriles 1. ${ }^{1 a}$ These reactions were facilitated by the aminonitrile substituents. Further investigation shows that this effect may play an essential role in nucleophilic-type radical cyclisations of 2-cyano 3-substituted indoles 3 to give spiro-annelated indolines $4^{1 b}$<smiles>[Y]CC(C)(C)CCC([R])/C=C(/C#N)Nc1ccccc1</smiles>

1<smiles>Cn1c(CCCCCBr)c(C#N)c2ccccc21</smiles>

3

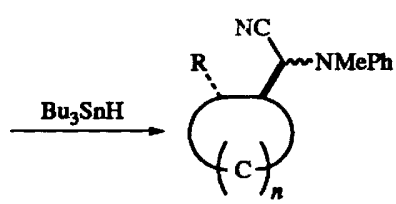

2

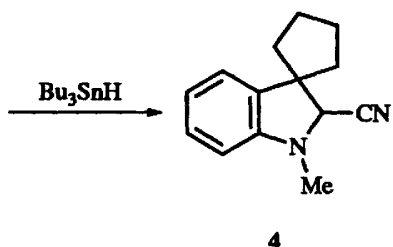

We report herein the preparation of $2-(N$-methylanilino $)-$ alka-2,5-dienenitriles 5-16 and their intramolecular radical cyclisations. These compounds have two alkenyl groups orientated in equal proximity to the radical centres. One alkenyl group contains both the cyano and $\mathrm{N}$-methylanilino substituents, while the other contains either an electronwithdrawing or electron-donating group. The radical cyclisation may occur in two competitive pathways, with attack at C-3 (path a in Scheme 1) or attack at C-5 (path b). The observed regioselectivity can thus reflect the influence of amino-cyano substitution in the free-radical cyclisations.

\section{Results and discussion}

The dienenitrile 5 was treated with $\mathrm{Bu}_{3} \mathrm{SnH}$ (1.2 equiv.) and a radical initiator azoisobutyronitrile (AIBN) (0.2 equiv.) in refluxing deoxygenated anhydrous benzene to effect the intramolecular free-radical cyclisation, giving 17a and 17b (34:66) in $77 \%$ yield. Both 17a and $17 \mathbf{b}$ had the trans configuration since they were derived from an exo-transition state $\mathbf{B}$ (Scheme 1). ${ }^{1 a, 2}$ Although the starting material 5 generally existed as a mixture of $2 E$ - and $2 Z$-isomers, the subsequent radical cyclisation gave the same ratio of dia-
Table 1 Free-radical cyclisations of 2-aminoalka-2,5-dienenitriles ( 1.2 equiv. of $\mathrm{Bu}_{3} \mathrm{SnH}, 0.2$ equiv. of $\mathrm{AIBN}, \mathrm{PhH}, 80^{\circ} \mathrm{C}, 6 \mathrm{~h}$ )

\begin{tabular}{ll} 
Substrate & Products (yield, \%; ratio of isomers) \\
\hline $\mathbf{5}$ & $\mathbf{1 7}(77 ; \mathbf{a}: \mathbf{b}=34: 66)$ \\
$\mathbf{6}$ & $\mathbf{1 8}(69 ; \mathbf{a}: \mathbf{b}=37: 63)$ \\
7 & $\mathbf{1 9}(87 ; \mathbf{a}: \mathbf{b}: \mathbf{c}: \mathbf{d}=39: 29: 25: 7)$ \\
$\mathbf{8}$ & $\mathbf{2 0}(82 ; \mathbf{a}: \mathbf{b}=40: 60)$ \\
$\mathbf{9}^{a}$ & $\mathbf{2 1}(38 ; \mathbf{a}: \mathbf{b}=54: 46)+\mathbf{2 2}(60 ; \mathbf{a}: \mathbf{b}=61: 39)$ \\
$\mathbf{1 0}$ & $\mathbf{2 3}(82 ; \mathbf{a}: \mathbf{b}=63: 37)$ \\
$\mathbf{1 1}$ & $\mathbf{2 4}(81 ; \mathbf{a}: \mathbf{b}: \mathbf{c}=20: 69: 11)$ \\
$\mathbf{1 2}$ & $\mathbf{2 5}(89 ; \mathbf{a}: \mathbf{b}=54: 46)$ \\
$\mathbf{1 3}$ & $\mathbf{2 6}(75 ; \mathbf{a}: \mathbf{b}: \mathbf{c}: \mathbf{d}=25: 10: 52: 23)+\mathbf{2 7}(19)$ \\
$\mathbf{1 4}$ & $\mathbf{2 8}(62 ; \mathbf{a}: \mathbf{b}=55: 45)$ \\
$\mathbf{1 5}$ & $\mathbf{2 9}(88 ; \mathbf{a}: \mathbf{b}=12: 88)$ \\
$\mathbf{1 6}$ & $\mathbf{3 0}(40 ; \mathbf{a}: \mathbf{b}: \mathbf{c}=55: 25: 20)$
\end{tabular}

${ }^{a}$ The reaction was carried out in the presence of 1.2 equiv. of AIBN.

stereoisomers $17 \mathbf{a}$ and $\mathbf{1 7 b}$ differing in their C-2 chirality. The structures of $17 \mathrm{a}$ and $17 \mathrm{~b}$ were tentatively assigned as such by analogy to those of 21a and 21b (see below). The less polar isomer 17a exhibited smaller chemical shifts for the $2-\mathrm{H}$ and C-2 signals than those of $17 \mathbf{b}$ (Table 2).

The intramolecular radical cyclisations of other dienenitriles, having methyl, phenyl, benzyloxy, phenylsulfanyl, methoxycarbonyl or cyano substituents at C-6, were similarly carried out (Table 1), whereas the reaction of 9 was conducted in the presence of an excess of AIBN (1.2 equiv.). The phenyl radical generated from 9 attacked exclusively at C-3 to give $38 \%$ of 21 $(\mathbf{a} / \mathbf{b}=54: 46)$ and $60 \%$ of $22(a / b=61: 39)$ by trapping the intermediate amino-cyano $\alpha$-radical with a hydrogen atom or a dimethylacetonitrile radical. All the cyclisation products had a trans configuration. Compounds 21b and 22b had the $\left(2 R S, 1^{\prime} R S, 2^{\prime} R S\right)$-structures as shown by the $\mathrm{X}$-ray diffraction analyses (Table 3 ). The atomic coordinates together with the bond lengths and bond angles for these crystallographic studies have been deposited with the Cambridge Crystallographic Data Centre. $\dagger$

A study of the reactions with alkyl, vinyl or phenyl radicals (Table 1), showed that most of the radical cyclisations occurred exclusively at C-3 (path a). Free-radical cyclisations of the dienenitrile 13 with a methoxycarbonyl group at C-6 occurred, however, at both C-3 and C-5 to give 26 and 27 (4:1). When two stereoisomers were obtained in individual cyclisation, the less polar isomer consistently had $2-\mathrm{H}$ and $\mathrm{C}-3$ resonances at higher

$\dagger$ For details, see 'Instructions for Authors (1995)', J. Chem. Soc., Perkin Trans. 1, 1995, Issue 1. 


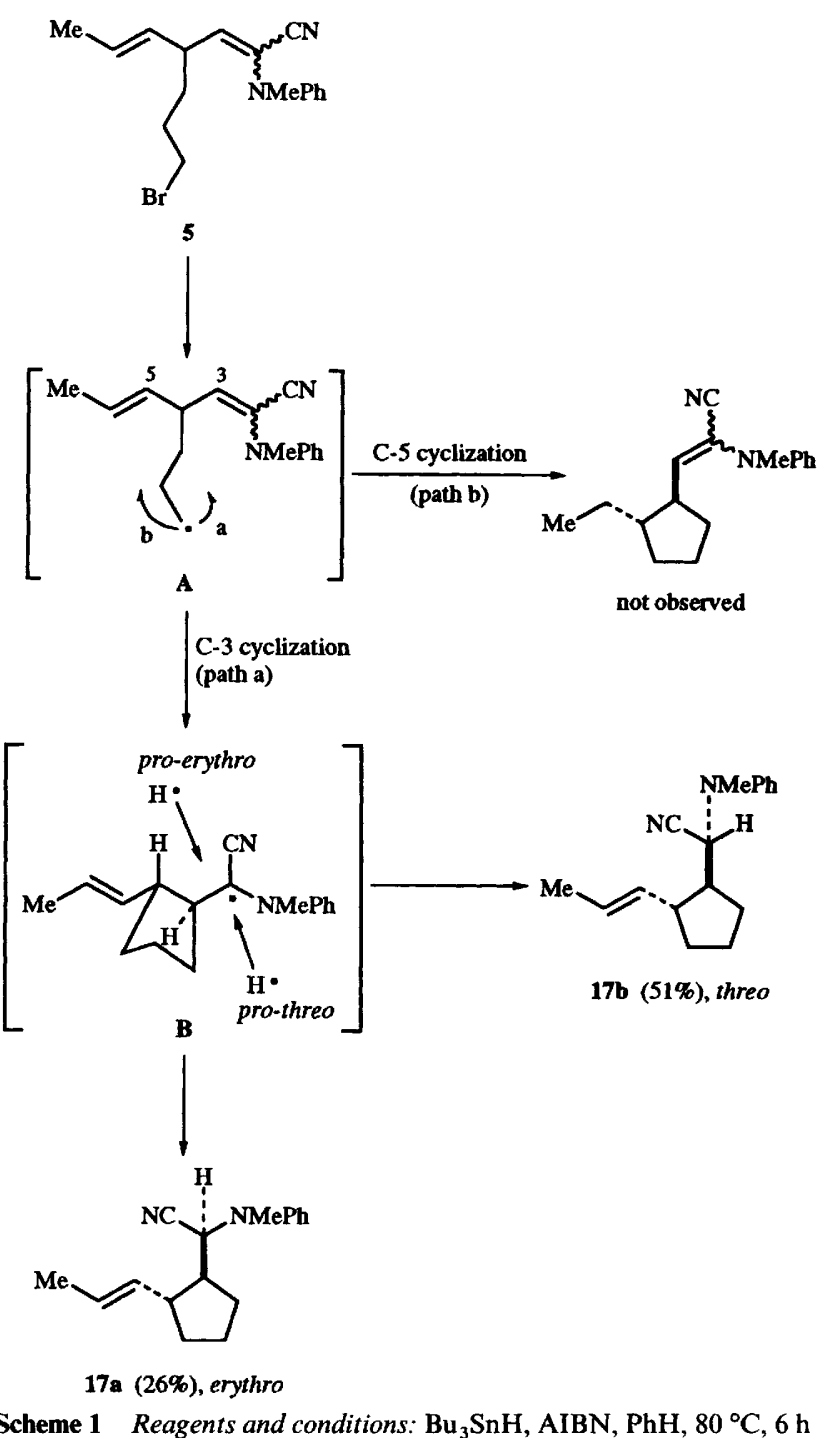

Table 2 Comparison of $2-\mathrm{H}$ and $\mathrm{C}-2$ signals in the isomers of cyclisation products $\left(300 \mathrm{MHz}, \mathrm{CDCl}_{3}, \mathrm{TMS}\right)$

\begin{tabular}{llllll}
\hline $\begin{array}{l}\text { Less polar } \\
\text { isomer }\end{array}$ & $\delta_{\text {2-H }}$ & $\delta_{\mathrm{C}-\mathbf{2}}$ & $\begin{array}{l}\text { More polar } \\
\text { isomer }\end{array}$ & $\delta_{2-\mathbf{H}}$ & $\delta_{\mathrm{C}-\mathbf{2}}$ \\
\hline 17a & 4.15 & 57.5 & $\mathbf{1 7 b}$ & 4.49 & 58.2 \\
$\mathbf{1 8 a}$ & 4.22 & 58.0 & $\mathbf{1 8 b}$ & $\mathbf{4 . 2 6}$ & 58.3 \\
20a & 4.22 & 57.4 & $\mathbf{2 0 b}$ & 4.33 & 57.8 \\
21a & 4.24 & 57.9 & $\mathbf{2 1 b}$ & 4.42 & 58.9 \\
23a & 4.37 & 58.0 & 23b & 4.49 & 58.8 \\
24a $(Z)$ & 4.56 & 57.4 & $\mathbf{2 4 b}(Z)$ & 4.77 & 58.1 \\
25a & 4.29 & 58.1 & $\mathbf{2 5 b}$ & 4.47 & 59.0 \\
26a $(E)$ & 4.37 & 57.9 & 26b $(E)$ & 4.42 & 59.1 \\
26c $(Z)$ & 4.39 & 58.0 & $\mathbf{2 6 d}(Z)$ & 4.68 & 58.9 \\
\hline
\end{tabular}

fields (Table 2). These results of regioselective cyclisations indicated that the aminonitrile substituents enhanced the reaction rates, either by the polar effects or by stabilising

$\ddagger$ The rate of cyclisation of hex-5-enyl radical is greatly enhanced by introduction of a cyano substituent at C-6. 6-Cyano-6-methoxyhex-5enyl radical cyclises slightly faster than 6-cyanohex-5-enyl radical. ${ }^{3 a, b}$ The nucleophilic alkyl radical is considered to add more rapidly to the electrophilic alkenenitrile due to the electron-withdrawing effect of the cyano group. Alternatively, the $\pi$-electron-accepting cyano group may increase exothermicity of the radical cyclisation by stabilising the intermediate $\alpha$-radical and thus facilitates the radical reaction. For detailed discussion, see ref. $3 c$.

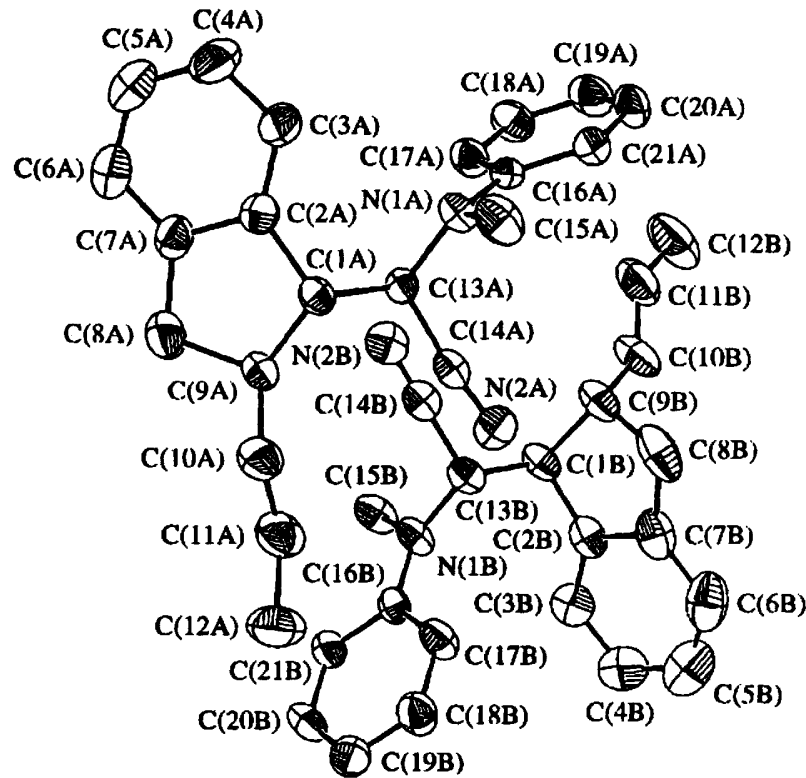

Fig. 1 ORTEP drawing of $21 b$

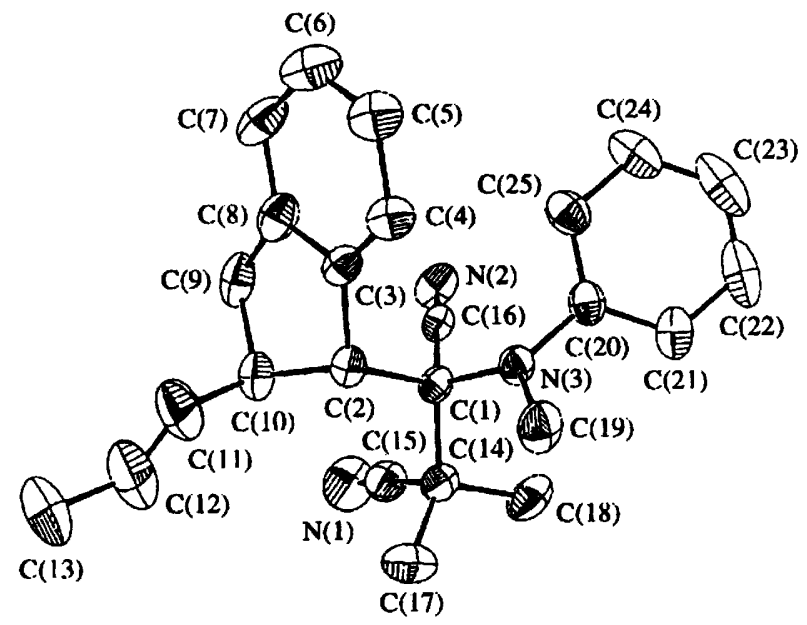

Fig. 2 ORTEP drawing of $\mathbf{2 2 b}$

the intermediate $\alpha$-radicals. $\ddagger$ The radical cyclisation of 4-(2-bromobenzyl-3,5-dimethyl-2-( $N$-methylanilino)hepta-2,5diene-1,7-dinitrile 14 also occurred exclusively at C-3. Such great preference for C-3 attack may be a result of the 2,3-double bond having more substituents than the 5,6-double bond. ${ }^{4}$ The electron-donating amino group may also exert a synergistic effect with the electron-withdrawing cyano group in stabilising the intermediate $\alpha$-radicals. ${ }^{3 a, 5}$

The amino nitrile 15 underwent the free-radical cyclisation efficiently to form the cyclohexanes 29 in $88 \%$ yield. The freeradical reaction of $\mathbf{1 6}$ gave the cyclohexenes 30 , presumably via the mechanism shown in Scheme 2. The radical $\mathbf{C}$ generated from 16 cyclised to give an unstable vinylcyclobutane intermediate $\mathbf{D}$, which ruptured to afford the amino-cyanosubstituted hept-6-enyl radicals $\mathbf{E}$ and subsequently recyclised to the observed products 30 .

Preparation of 2-( $\boldsymbol{N}$-methylanilino)alka-2,5-dienenitriles

Condensation of hexa-2,4-dienal, potassium cyanide and $N$-methylaniline according to Strecker's method gave 2-( $N$-methylanilino $)$ hepta-3,5-dienenitrile, ${ }^{6}$ which underwent regioselective $\gamma$-alkylations with dihalides to give the hepta2,5-dienenitriles 5-9, 15 and 16 predominating in the $2 Z$ - 
<smiles>C=CC(C=C(C#N)NCCCCBr)CCCBr</smiles>

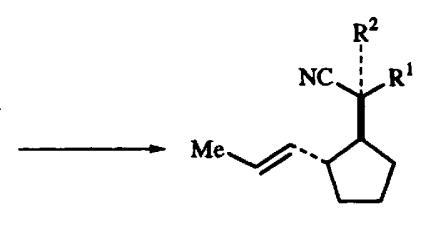

17a $R^{1}=N M e P h, R^{2}=H$ 17b $R^{1}=H, R^{2}=$ NMePh<smiles>CC=CC(C=C(C#N)NCCCC)CC=CBr</smiles><smiles>[R]C([R])([R])C(C#N)C1C=CCC1C=CC</smiles>

6

18a $R^{1}=\mathrm{NMePh}, \mathrm{R}^{2}=\mathrm{H}$ $18 b R^{1}=H, R^{2}=N M e P h$<smiles>CC=CC(C=C(C#N)NCCCCC(C)Br)CCC(C)Br</smiles><smiles>CC=CC1CCC(C)C1C(C#N)C(C#N)NCC</smiles>

19<smiles>C/C=C/C(/C=C(/C#N)N(C)C)CC1=C(Br)CCC1</smiles>

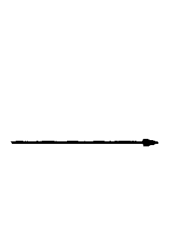

(n)

20a $R^{1}=\mathrm{NMePh}, \mathrm{R}^{2}=\mathrm{H}$ 20b $R^{1}=H, R^{2}=N M e P h$<smiles>CC=CC(C=C(C#N)NCCC)Cc1ccccc1Br</smiles><smiles>[R]C([R])([X])C1c2ccccc2CC1/C=C/C</smiles>

21a $R^{1}=\mathrm{NMePh}, \mathrm{R}^{2}=\mathrm{H}$ 21b $R^{1}=H, R^{2}=N M e P h$ 22a $\mathrm{R}^{1}=\mathrm{Me}_{2} \mathrm{C}(\mathrm{CN}), \mathrm{R}^{2}=\mathrm{NMePh}$ 22b $R^{1}=\mathrm{NMePh}, \mathrm{R}^{2}=\mathrm{Me}_{2} \mathrm{C}(\mathrm{CN})$<smiles>CN(C)C(C#N)=CC(C=Cc1ccccc1)Cc1ccccc1Br</smiles>

10<smiles>[X][W]#[W]</smiles>

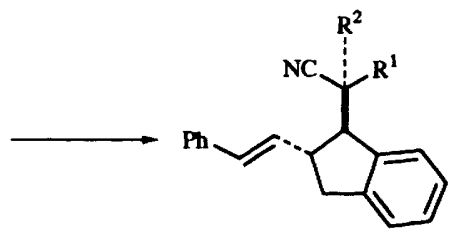

23a $R^{1}=N M e P h, R^{2}=H$ 23b $R^{1}=H, R^{2}=N M e P h$<smiles>[R]C([R])(C#N)C1CCCC1/C=C\Oc1ccccc1</smiles>

24a (Z) $R^{1}=\mathrm{NMePh}, \mathrm{R}^{2}=\mathrm{H}$ 24b (Z) $R^{1}=H, R^{2}=N M e P h$ 24c $(E) \mathrm{R}^{1}=\mathrm{H}, \mathrm{R}^{2}=\mathrm{NMePh}$<smiles>CN(C)/C(C#N)=C/C(/C=C/[SnH+])Cc1ccccc1Br</smiles>

12

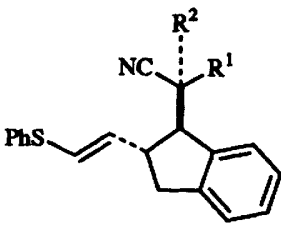

25a $R^{1}=N M e P h, R^{2}=H$ 25b $R^{1}=H, R^{2}=N M e P h$

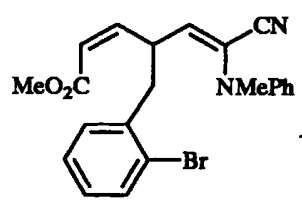

13

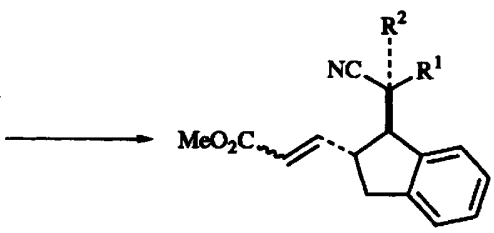

26a (E) $\mathrm{R}^{1}=\mathrm{NMePh}, \mathrm{R}^{2}=\mathrm{H}$ 26b (E) $R^{1}=H, R^{2}=N M e P h$ $26 c(Z) R^{1}=\mathrm{NMePh}, R^{2}=H$ 26d (Z) $R^{1}=H, R^{2}=N M e P h$<smiles>CC(=O)CC1c2ccccc2CC1/C=C(\C#N)Nc1ccccc1</smiles>

27
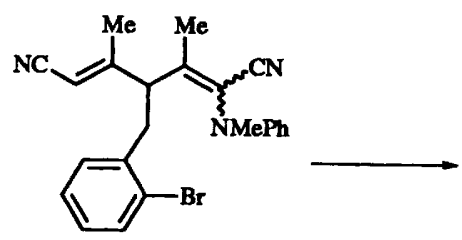<smiles>[Y4]C(C)=CC1Cc2ccccc2C1(C)C([R])([R])C</smiles>

14

28a $R^{1}=N M e P h, R^{2}=H$ 28b $R^{1}=H, R^{2}=N M e P h$<smiles>C/C=C/C(/C=C(/C#N)Nc1ccccc1)CCCCBr</smiles>

15<smiles>[M]C=CC1CCCCC1C([R])([R])C#N</smiles>

29. $R^{1}=N M e P h, R^{2}=H$ 29b $R^{1}=H, R^{2}=N M e P b$<smiles>C/C=C/C(/C=C(/C#N)NCCCCBr)CCBr</smiles><smiles>C[C@@H]1C=CCC[C@H]1C(C#N)N(C)C</smiles> 
Table 3 Crystal data for (2RS, 1' $\left.R S, 2^{\prime} R S\right)$-2-(2,3-dihydro-2-prop-1-enyl-1 $H$-indan-1-yl)-2-( $N$-methylanilino)acetonitrile $21 \mathbf{b}$ and ( $\left.2 R S, 1^{\prime} R S, 2^{\prime} R S\right)$ 2-(2,3-dihydro-2-prop-1-enyl-1 $H$-indan-1-yl)-3,3-dimethyl-2-( $N$-methylanilino)butane-1,4-dinitrile 22b. The X-ray data were collected on a CAD-4 diffractometer. The analyses were carried out on a microVAX III computer using NRC SDP software.

\begin{tabular}{lll}
\hline & 21b & 22b \\
\hline Formula & $\mathrm{C}_{21} \mathrm{H}_{22} \mathrm{~N}_{2}$ & $\mathrm{C}_{25} \mathrm{H}_{27} \mathrm{~N}_{3}$ \\
Space group & $P_{-1} P_{21 / \mathrm{c}}$ & $10.388(2)$ \\
$a / \AA$ & $11.013(3)$ & $20.755(5)$ \\
$b / \AA$ & $11.847(3)$ & $10.633(2)$ \\
$c / \AA$ & $14.212(3)$ & 90 \\
$x /{ }^{\circ}$ & $87.68(2)$ & $109.76(1)$ \\
$\beta /{ }^{\circ}$ & $93.23(2)$ & 90 \\
$\gamma /{ }^{\circ}$ & $106.16(2)$ & $2292.5(3)$ \\
$V / \AA^{3}$ & $1854.2(3)$ & $0.4 \times 0.2 \times 0.1$ \\
Crystal size (mm) & $0.3 \times 0.2 \times 0.1$ & 4 \\
$Z$ & 4 & 298 \\
$T / \mathrm{K}$ & 298 & $\mathrm{Mo-K} \alpha(=0.7107 \AA)$ \\
Radiation & $\mathrm{Mo-K} \alpha(=0.7107 \AA)$ & $2-45$ \\
$2 \theta_{\text {max }}{ }^{\circ}$ & $2-45$ & $1.648-8.24$ \\
Scan speed (deg min $\left.{ }^{-1}\right)$ & $1.618-8.24$ & $2(0.65+0.35 \tan \theta)$ \\
Scan parameters & $2(0.70-0.35 \tan \theta)$ & 2805 \\
No. of measurements & 4636 & $1350(>2 \sigma)$ \\
No. of observed reflections & $2150(>2 \sigma)$ & 0.052 \\
$R$ & 0.054 & 0.054 \\
$R_{\mathrm{w}}$ & 0.056 & 2.02 \\
$S$ & 2.12 & \\
\hline
\end{tabular}

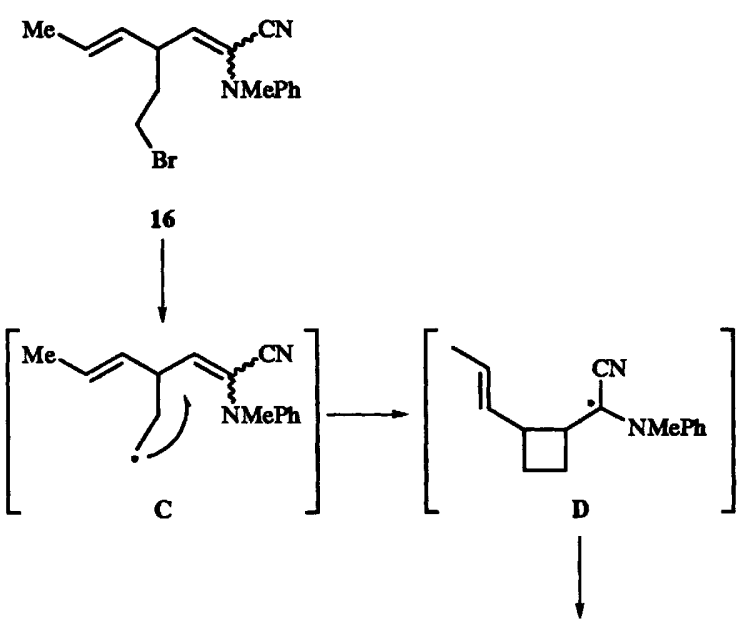

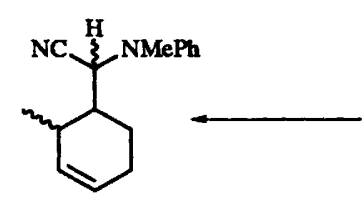

30

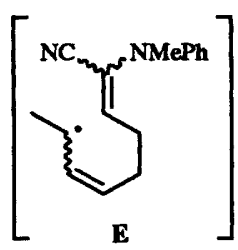

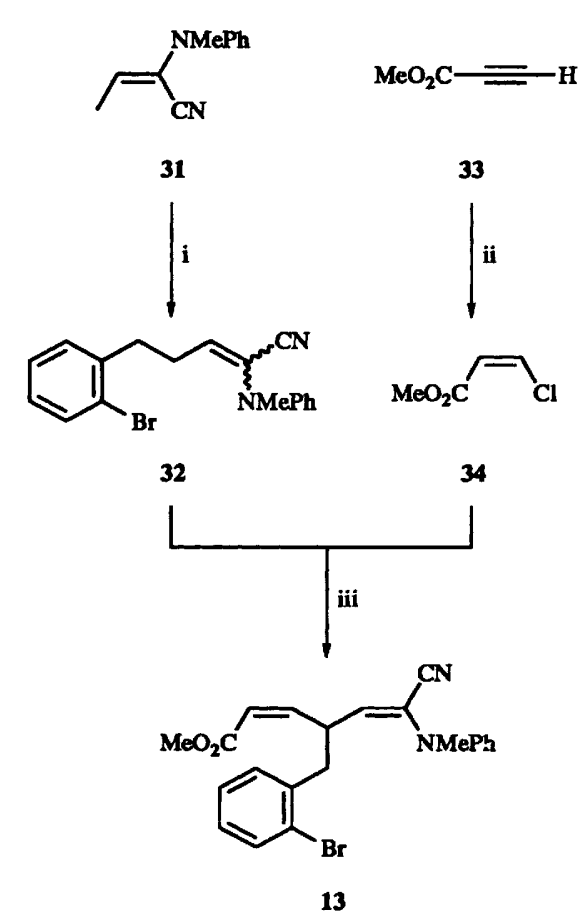

Scheme 2 Reagents and conditions: $\mathrm{Bu}_{3} \mathrm{SnH}, \mathrm{AIBN}, \mathrm{PhH}, 80^{\circ} \mathrm{C}, 6 \mathrm{~h}$

configuration. ${ }^{7}$ By similar procedures, the hexadienenitriles 10 12 having phenyl, benzyloxy or a phenylsulfanyl substituent at C-6 were prepared by $\gamma$-alkylations of appropriate aminocyanopentadienyl anions. ${ }^{7}$ Compound 13 was prepared by condensation of 5-(2-bromophenyl)-2-( $N$-methylanilino)pent2-enenitrile 32 with methyl 3-chloroacrylate (Scheme 3). Acetylation of 3-methyl-2-( $N$-methylanilino)but-2-enenitrile 36, followed by alkylation with 2-bromobenzyl bromide, afforded a 5-oxo-2-amino nitrile 37 (Scheme 4). Subsequent Wittig-Horner reaction of 37 with diethyl cyanomethylphosphonate resulted in the desired heptadienedinitrile 14.

4-(2-Bromobenzyl)-6-cyano-2-( $N$-methylanilino)hepta-2,5diene-1,7-dinitrile $\mathbf{F}$ is an ideal substrate for the study of the captodative effect in free-radical cyclisations. ${ }^{5}$ Attempts to prepare this substrate, either from 9 or from 32, however, failed (Scheme 5). Ozonolysis of 9 occurred selectively at the 2,3-

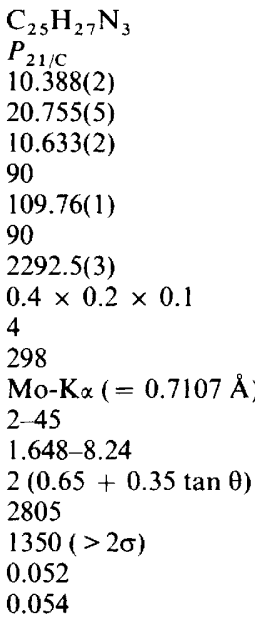

0.052

0.05 


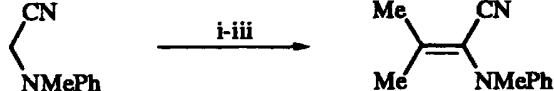

35

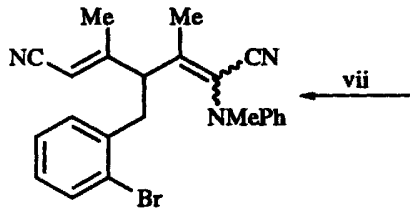

14
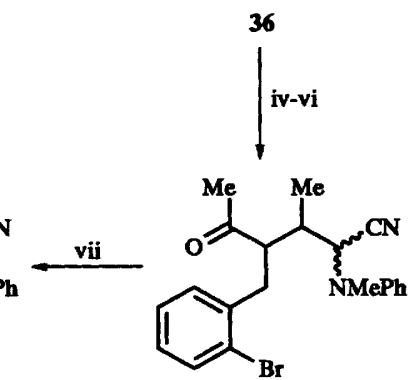

37

Scheme 4 Reagents and conditions: i, LDA, THF; $\mathrm{Me}_{2} \mathrm{CO},-78^{\circ} \mathrm{C}$; ii, $\mathrm{Ac}_{2} \mathrm{O}$, pyridine, $\mathrm{CH}_{2} \mathrm{Cl}_{2}$; iii, $\mathrm{Bu}^{t} \mathrm{OK}, \mathrm{THF},-78^{\circ} \mathrm{C} ; 71 \%$; iv, LDA, THF; $\mathrm{Ac}_{2} \mathrm{O} ; \mathrm{v}, \mathrm{MeONa}, \mathrm{MeOH}, 25^{\circ} \mathrm{C}, 10 \mathrm{~min}$; vi, LDA, THF, HMPA; $o-\mathrm{BrC}_{6} \mathrm{H}_{4} \mathrm{CH}_{2} \mathrm{Br},-78^{\circ} \mathrm{C}$ to $25^{\circ} \mathrm{C} ; 47^{\circ}$; vii, $\mathrm{NCCH}_{2} \mathrm{P}(\mathrm{O})(\mathrm{OEt})_{2}$, $\mathrm{NaH}, \mathrm{THF},-78$ to $25^{\circ} \mathrm{C} ; 82 \%$

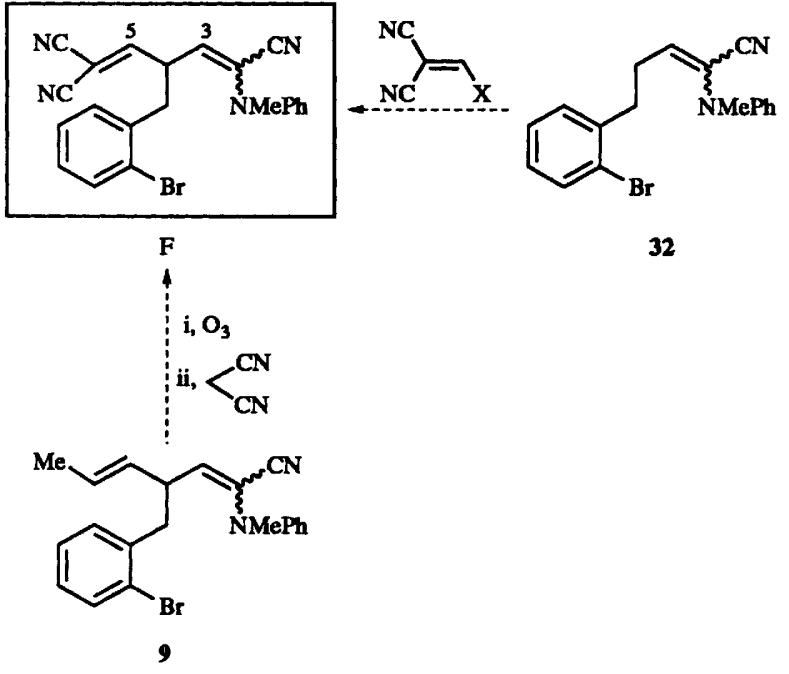

Scheme 5

amines, amides, carbonyl compounds and $\alpha$-amino acids, $\S$ the present method can be useful in the preparation of functionalized cyclopentanes, cyclopentenes, cyclohexanes, indanes and bicyclo[3.3.0]

\section{Experimental}

Mps are uncorrected. ${ }^{1} \mathrm{H}$ NMR spectra were recorded at 200 or $200 \mathrm{MHz}$ and ${ }^{13} \mathrm{C}$ NMR 50 or $75 \mathrm{MHz}$ using chlorotrimethylsilane as an internal standard ( $J$ values in $\mathrm{Hz}$ ). Mass spectra (using a Finnigan TSQ 46C spectrometer) were recorded at an ionizing voltage of $70 \mathrm{eV}$. Merck silica gel $60 \mathrm{~F}$ sheets were used for analytical thin-layer chromatography. Column chromatography was performed on $\mathrm{SiO}_{2} \quad(70-230$ mesh); gradient of EtOAc and hexane were used as eluents. High-pressure liquid chromatography was carried out on a liquid chromatograph equipped with a refractive index detector. The samples were analysed and/or separated on Hibar Lichrosorb Si60 $(7 \mu \mathrm{m})$ column $(25 \times 1 \mathrm{~cm})$ with the indicated

$\S$ For use of $x$-amino nitriles in organic synthesis, such as the acid- or base-catalysed hydrolyses of $\alpha$-amino nitriles to carbonyl compounds and $x$-amino acids, see the review in ref. $8 a$. For reductive decyanation and alkylative decyanation of $\alpha$-amino nitriles to amines, see ref. $8 b$. For oxidative conversion of $\alpha$-amino nitriles to amides, see refs. $8 c$ and $8 d$

eluent with a $5 \mathrm{~cm}^{3} \min ^{1}$ flow rate. Benzene and THF were distilled from sodium benzophenone ketyl under $\mathrm{N}_{2}$. The X-ray diffraction data were collected on a CAD- 4 diffractometer. The analyses were carried out on a microVAX III computer using NRC SDP software.

Compounds 5-12, 15 and 16 were prepared by reported procedures. ${ }^{6.7}$

Methyl 4-(2-bromophenylmethyl)-6-cyano-6-( $N$-methylanilino)hexa-2,5-dienoate 13

Under a nitrogen atmosphere, a THF solution $\left(5 \mathrm{~cm}^{3}\right)$ of $5-$ (2-bromophenyl)-2-( $N$-methylanilino)pent-2-enenitrile 32 (300 $\left.\mathrm{mg}, 0.88 \mathrm{~cm}^{3}\right)$ was added dropwise to a cold $\left(-78^{\circ} \mathrm{C}\right) \mathrm{LDA}$ solution prepared from BuLi (1.6 mol dm $\mathrm{dm}^{-3}$ hexane solution; $\left.0.6 \mathrm{~cm}^{3}\right)$ and a THF solution $\left(10 \mathrm{~cm}^{3}\right)$ of diisopropylamine $\left(0.15 \mathrm{~cm}^{3}, 0.95 \mathrm{mmol}\right)$. The orange solution was stirred at $-78^{\circ} \mathrm{C}$ for $45 \mathrm{~min}$, after which a THF solution $\left(5 \mathrm{~cm}^{3}\right)$ of methyl 3-chloroacrylate ${ }^{8}(130 \mathrm{mg}, 1.1 \mathrm{mmol})$ was added to it; the colour faded immediately and the mixture was warmed to $25^{\circ} \mathrm{C}$. Saturated aqueous $\mathrm{NH}_{4} \mathrm{Cl}$ was added to the mixture and the THF was removed under reduced pressure. The residue was extracted with EtOAc $\left(6 \mathrm{~cm}^{3} \times 5\right)$ and the combined extracts were washed with brine, dried $\left(\mathrm{Na}_{2} \mathrm{SO}_{4}\right)$, filtered and concentrated. Chromatography of the residue on a silica-gel column with EtOAc-hexane $(10: 90)$ as eluent gave the title compound $13(285 \mathrm{mg}, 77 \%)$ of $2 Z, 5 Z$-configuration as a yellow oil; TLC [EtOAc-hexane $(10: 90)] R_{\mathrm{F}} 0.14 ; v_{\max }($ neat $) / \mathrm{cm}^{-1}$ $2227(\mathrm{CN})$ and $1721(\mathrm{CO}) ; \delta_{\mathrm{H}}\left(\mathrm{CDCl}_{3}\right) 3.02(2 \mathrm{H}, \mathrm{dd}, J 8,2)$, $3.03(3 \mathrm{H}, \mathrm{s}, \mathrm{NMe}), 3.75(3 \mathrm{H}, \mathrm{s}, \mathrm{OMe}), 4.96-5.13(1 \mathrm{H}, \mathrm{m}, 4-\mathrm{H})$, $5.56(1 \mathrm{H}, \mathrm{d}, J 10,5-\mathrm{H}), 5.83(1 \mathrm{H}, \mathrm{d}, J 12,2-\mathrm{H}), 6.12(1 \mathrm{H}, \mathrm{dd}, J$ $12,10,3-\mathrm{H}), 6.76(2 \mathrm{H}, \mathrm{d}, J 8), 7.00(1 \mathrm{H}, \mathrm{ddd}, J 8,8,1), 7.08-7.30$ $(4 \mathrm{H}, \mathrm{m}), 7.37(1 \mathrm{H}$, ddd, $J 8,8,1)$ and $7.56(1 \mathrm{H}, \mathrm{d}, J 8)$; $\delta_{\mathrm{C}}\left(\mathrm{CDCl}_{3}\right) 40.0(\mathrm{q}), 40.7(\mathrm{t}), 40.8(\mathrm{q}), 51.5(\mathrm{~d}), 113.9(\mathrm{~s}, \mathrm{CN})$, 120.4 (d), 120.7 (d, 2 C), 122.8 (s, C-6), 123.1 (d), 125.0 (s), 127.5 (d), 127.7 (d), 128.2 (d), 129.0 (d, 2 C), 131.8 (d), 132.6 (d), 137.4 (s), 146.1 (s), 146.8 (d, C-5) and 165.9 (s, C-1); $m /=426(3 \%$, $\left.[\mathrm{M}+2]^{+}\right), 424\left(3, \mathrm{M}^{+}\right)$and $255(100)$ (Found: $\mathrm{M}^{+}, 426.0755$. Calc. for $\left.\mathrm{C}_{22} \mathrm{H}_{21}{ }^{81} \mathrm{BrN}_{2} \mathrm{O}_{2}: M, 426.0767\right)$.

\section{4-(2-Bromophenylmethyl)-3,5-dimethyl-2-( $N$-methylanilino)- hepta-2,5-diene-1,7-dinitrile 14}

Under a nitrogen atmosphere, a THF solution $\left(5 \mathrm{~cm}^{3}\right)$ of $3-$ methyl-2- $(N$-methylanilino $)$ but-2-enenitrile $(0.94 \mathrm{~g}, 5.1 \mathrm{mmol})$ was added dropwise to a cold $\left(-78^{\circ} \mathrm{C}\right)$ LDA solution prepared from BuLi (1.6 mol dm$m^{-1}$ hexane solution; $\left.3.6 \mathrm{~cm}^{3}\right)$ and a THF solution $\left(10 \mathrm{~cm}^{3}\right)$ of diisopropylamine $\left(0.90 \mathrm{~cm}^{3}, 6.9 \mathrm{mmol}\right)$. The orange solution was stirred for $45 \mathrm{~min}$ after which acetic anhydride $\left(0.72 \mathrm{~cm}^{3}, 7.1 \mathrm{mmol}\right)$ was added to it and the mixture was warmed to $25^{\circ} \mathrm{C}$. Saturated aqueous $\mathrm{NH}_{4} \mathrm{Cl}$ was added to the mixture from which THF was removed under reduced pressure. The residue was extracted with EtOAc $\left(5 \mathrm{~cm}^{3} \times 4\right)$ and the combined extracts were washed with brine, dried $\left(\mathrm{Na}_{2} \mathrm{SO}_{4}\right)$, filtered and concentrated. Chromatography of the residue on a silica gel column with EtOAc-hexane $(5: 95)$ as eluent gave 5-acetoxy-3-methyl-2-( $N$-methylanilino)hexa-2,4dienenitrile $(1.01 \mathrm{~g}, 74 \%)$. The product $(900 \mathrm{mg}, 3.3 \mathrm{mmol})$ was saponified by MeONa (180 mg, $3.3 \mathrm{mmol})$ in $\mathrm{MeOH}\left(10 \mathrm{~cm}^{3}\right)$ at $25^{\circ} \mathrm{C}$ for $10 \mathrm{~min}$ to give 3 -methyl-2-( $N$-methylanilino $)-5$ oxohex-2-enenitrile $(650 \mathrm{mg}, 86 \%)$.

A THF solution $\left(5 \mathrm{~cm}^{3}\right)$ of 3-methyl-2-( $N$-methylanilino)-5oxohex-2-enenitrile ( $385 \mathrm{mg}, 1.7 \mathrm{mmol}$ ) was added dropwise to a cold $\left(-78^{\circ} \mathrm{C}\right)$ LDA solution prepared from BuLi $(1.6 \mathrm{~mol}$ $\mathrm{dm}^{-1}$ hexane solution; $\left.1.2 \mathrm{~cm}^{3}\right)$ and a THF solution $\left(10 \mathrm{~cm}^{3}\right)$ of diisopropylamine $\left(0.34 \mathrm{~cm}^{3}, 2.0 \mathrm{mmol}\right)$. The brown solution was stirred for $45 \mathrm{~min}$ after which a THF solution $\left(5 \mathrm{~cm}^{3}\right)$ of $2-$ bromobenzyl bromide ( $500 \mathrm{mg}, 2.0 \mathrm{mmol}$ ) was added dropwise to it; the mixture was then warmed to $25^{\circ} \mathrm{C}$. Saturated aqueous $\mathrm{NH}_{4} \mathrm{Cl}$ was added to the mixture from which THF was removed 
under reduced pressure. The residue was extracted with EtOAc $\left(5 \mathrm{~cm}^{3} \times 4\right)$ and the combined extracts were washed with brine, dried $\left(\mathrm{Na}_{2} \mathrm{SO}_{4}\right)$, filtered and concentrated. Chromatography of the residue on a silica-gel column with EtOAc-hexane (10:90) as eluent to give the alkylation product $37(590 \mathrm{mg}, 73 \%)$ containing $E$ - and $Z$-isomers $(54: 46)$.

To a cold $\left(-78^{\circ} \mathrm{C}\right)$ THF suspension $\left(10 \mathrm{~cm}^{3}\right)$ of $\mathrm{NaH}$ (dispersed in $60 \%$ mineral oil; $30 \mathrm{mg}, 1.1 \mathrm{mmol}$ ) was added diethyl cyanomethylphosphonate $\left(0.13 \mathrm{~cm}^{3}, 0.8 \mathrm{mmol}\right)$. The mixture was stirred for $45 \mathrm{~min}$ after which, a THF solution $\left(5 \mathrm{~cm}^{3}\right)$ of $37(230 \mathrm{mg}, 0.58 \mathrm{mmol})$ was added dropwise to it. The mixture was warmed to $25^{\circ} \mathrm{C}$ and stirred for $24 \mathrm{~h}$. Saturated aqueous $\mathrm{NH}_{4} \mathrm{Cl}$ was added to the mixture from which THF was removed under reduced pressure. The residue was extracted with EtOAc $\left(6 \mathrm{~cm}^{3} \times 5\right)$ and the combined extracts were washed with brine, dried $\left(\mathrm{Na}_{2} \mathrm{SO}_{4}\right)$, filtered and concentrated. Chromatography of the residue on a silica-gel column with EtOAc-hexane (10:90) as eluent gave (2E,5E)-14 (100 mg, 41\%) and $(2 Z, 5 E)-14(100 \mathrm{mg}, 41 \%)$ accompanied by recovery of 37 (56 mg).

(2E,5E)-14: white solid, mp $131-133^{\circ} \mathrm{C}$; TLC [EtOAchexane $(10: 90)] \quad R_{\mathrm{F}} \quad 0.11 ; \quad v_{\max }(\mathrm{KBr}) / \mathrm{cm}^{-1} 2218 \quad(\mathrm{CN})$; $\delta_{\mathrm{H}}\left(\mathrm{CDCl}_{3}\right) 1.72(3 \mathrm{H}, \mathrm{s}), 2.18(3 \mathrm{H}, \mathrm{s}), 2.86(3 \mathrm{H}, \mathrm{s}), 3.10(1 \mathrm{H}$, dd, $J 13.5,11), 3.25(1 \mathrm{H}, \mathrm{dd}, J 13.5,4.5), 4.24(1 \mathrm{H}, \mathrm{dd}, J 11,4.5$, 4-H), 5.44( $1 \mathrm{H}, \mathrm{s}, 6-\mathrm{H}), 6.28(2 \mathrm{H}, \mathrm{d}, J 8), 6.83(1 \mathrm{H}$, ddd, $J 8,8,1)$, $7.12-7.25(4 \mathrm{H}, \mathrm{m}), 7.34(1 \mathrm{H}, \mathrm{dd}, J 8,8)$ and $7.65(1 \mathrm{H}, \mathrm{dd}, J 8,1)$; $\delta_{\mathrm{C}}\left(\mathrm{CDCl}_{3}\right) 13.4(\mathrm{q}), 21.2(\mathrm{q}), 35.5(\mathrm{t}), 37.5(\mathrm{q}), 50.6(\mathrm{~d}, \mathrm{C}-4)$, $97.7(\mathrm{~d}), 113.8(\mathrm{~d}, 2 \mathrm{C}), 114.4(\mathrm{~s}), 116.2(\mathrm{~s}), 119.8(\mathrm{~d}), 120.1(\mathrm{~s})$, 124.8 (s), 127.8 (d), 129.0 (d), 129.2 (d, 2 C), 130.9 (d), 133.5 (d), $136.2(\mathrm{~s}), 145.6(\mathrm{~s}), 150.8(\mathrm{~s})$ and $162.2(\mathrm{~s}) ; \mathrm{m} / z 421(8 \%), 419(8$, $\mathrm{M}^{+}$for ${ }^{79} \mathrm{Br}$ ) and $250(100)$. (2Z,5E)-14: yellow oil; TLC [EtOAc-hexane $(10: 90)] \quad R_{\mathrm{F}} 0.10 ; v_{\max }($ neat $) / \mathrm{cm}^{-1} 2249$ and $2217 ; \delta_{\mathrm{H}}\left(\mathrm{CDCl}_{3}\right) 1.96(3 \mathrm{H}, \mathrm{s}), 2.17(3 \mathrm{H}, \mathrm{s}), 2.46(3 \mathrm{H}, \mathrm{s}), 2.89$ $(1 \mathrm{H}, \mathrm{dd}, J 14,10), 3.14(1 \mathrm{H}, \mathrm{dd}, J 14,5.6), 4.28(1 \mathrm{H}, \mathrm{dd}, J 10$, 5.6), $5.15(1 \mathrm{H}, \mathrm{s}), 6.60(2 \mathrm{H}, \mathrm{d}, J 8), 6.88(1 \mathrm{H}, \mathrm{ddd}, J 8,8,1), 7.02$ $(1 \mathrm{H}, \mathrm{dd}, J 8,1), 7.11(1 \mathrm{H}$, ddd, $J 8,8,1), 7.18-7.26(3 \mathrm{H}, \mathrm{m})$ and $7.52(1 \mathrm{H}, \mathrm{dd}, J 8,1) ; \delta_{\mathrm{C}}\left(\mathrm{CDCl}_{3}\right) 16.4(\mathrm{q}), 21.2(\mathrm{q}), 36.3(\mathrm{t}), 38.7$ (q), 47.0(d), 98.4(d), 114.5(s), $115.0(\mathrm{~d}, 2 \mathrm{C}), 116.2(\mathrm{~s}), 120.0(\mathrm{~s})$, 120.5 (d), 124.8 (s), 127.8 (d), 129.0 (d), 129.3 (d, 2 C), 130.6 (d), 133.4 (d), 136.8 (s), 147.1 (s), 151.7 (s) and $162.4(\mathrm{~s}) ; m / z 422$ $(13 \%), 421(24), 420(13), 419\left(23, \mathrm{M}^{+}\right)$and $250(100)$ (Found: $\mathrm{M}^{+}, 419.1001$. Calc. for $\mathrm{C}_{23} \mathrm{H}_{22}{ }^{79} \mathrm{BrN}_{3}: M, 419.0998$ ).

\section{trans-2-( $N$-Methylanilino)-2-(prop-1-enylcyclopentyl)aceto- \\ nitrile 17}

Compound 5 [a mixture of $(2 E, 5 E)$ - and $(2 Z, 5 E)$-isomers $(40: 60), 200 \mathrm{mg}, 0.60 \mathrm{mmol}]$ in deoxygenated anhydrous benzene $\left(15 \mathrm{~cm}^{3}\right)$ was heated to reflux under an atmosphere of $\mathrm{N}_{2}$. A mixture of $\mathrm{Bu}_{3} \mathrm{SnH}\left(0.20 \mathrm{~cm}^{3}, 0.72 \mathrm{mmol}\right)$ and AIBN $(20 \mathrm{mg}, 0.12 \mathrm{mmol})$ in benzene $\left(15 \mathrm{~cm}^{3}\right)$ was added drop-bydrop at a rate of $0.1 \mathrm{~cm}^{3} \min ^{1}$ by means of a syringe pump. After completion of the addition, the reaction mixture was kept under reflux for $6 \mathrm{~h}$ and then cooled and concentrated under reduced pressure. The residue was chromatographed on a silicagel column with hexane as eluent to remove most of the tin compounds; subsequently elution with EtOAc gave the crude cyclization products 17 . The EtOAc solution was concentrated to $c a .10 \mathrm{~cm}^{3}$ and then treated with a small amount of $\mathrm{Et}_{3} \mathrm{~N}(0.5$ $\mathrm{cm}^{3}$ ) to precipitate out the residual tin compounds. The white precipitates were filtered off, and the filtrate was concentrated and chromatographed on a silica-gel column with EtOAchexane (2:98) as eluent to give $17(116 \mathrm{mg}, 77 \%)$ containing two isomers $(\mathbf{a}: \mathbf{b}=34: 66)$ which was separated by HPLC. 17a: White solid; mp $40-41^{\circ} \mathrm{C}$; TLC [EtOAc-hexane $\left.(2: 98)\right] R_{\mathrm{F}}$ $0.10 ; v_{\max }(\mathrm{KBr}) / \mathrm{cm}^{-1} 2214(\mathrm{CN}) ; \delta_{\mathrm{H}}\left(\mathrm{CDCl}_{3}\right) 1.46-1.72(4 \mathrm{H}$, $\mathrm{m}), 1.68(3 \mathrm{H}, \mathrm{d}, J 6), 1.72-1.92(2 \mathrm{H}, \mathrm{m}), 2.19-2.28(1 \mathrm{H}, \mathrm{m})$, $2.34-2.43(1 \mathrm{H}, \mathrm{m}), 2.87(3 \mathrm{H}, \mathrm{s}), 4.15(1 \mathrm{H}, \mathrm{d}, J 10,2-\mathrm{H}), 5.44$ $(1 \mathrm{H}, \mathrm{dd}, J 16,8), 5.58(1 \mathrm{H}, \mathrm{dq}, J 16,6), 6.90-6.93(3 \mathrm{H}, \mathrm{m})$ and
$7.28(2 \mathrm{H}, \mathrm{dd}, J 8,8) ; \delta_{\mathrm{C}}\left(\mathrm{CDCl}_{3}\right) 17.8(\mathrm{q}), 24.0(\mathrm{t}), 29.5(\mathrm{t}), 32.7$ (t), 35.1 (q), 47.2 (d), 47.3 (d), 57.5 (d, C-2), 115.6 (d, 2 C, C-2'), 117.3 (s, CN), 120.0 (d), 125.8 (d), 129.3 (d, 2 C, C-3'), 133.8 (d) and $149.1\left(\mathrm{~s}, \mathrm{C}-\mathrm{l}^{\prime}\right) ; m / z 254\left(14 \%, \mathrm{M}^{+}\right)$and $145(100)$ (Found: $\mathrm{M}^{+}, 254.1783$. Calc. for $\left.\mathrm{C}_{17} \mathrm{H}_{22} \mathrm{~N}_{2}: M, 254.1783\right)$. 17b: White solid, mp 107-109 ${ }^{\circ} \mathrm{C}$; TLC [EtOAc-hexane $(2: 98)$ ] $R_{\mathrm{F}} 0.08$; $v_{\max }(\mathrm{KBr}) / \mathrm{cm}^{-1} 2225(\mathrm{CN}) ; \delta_{\mathrm{H}}\left(\mathrm{CDCl}_{3}\right) 1.52-1.72(4 \mathrm{H}, \mathrm{m})$, $1.55(3 \mathrm{H}, \mathrm{dd}, J 7,2), 1.82-1.89(1 \mathrm{H}, \mathrm{m}), 1.92-2.01(1 \mathrm{H}, \mathrm{m})$, 2.09-2.14 (1 H, m), 2.26-2.32 (1 H, m), 2.65-2.74 (1 H, m), 2.88 $(3 \mathrm{H}, \mathrm{s}), 4.49(1 \mathrm{H}, \mathrm{d}, J 7, \mathrm{H}-2), 5.31(1 \mathrm{H}$, ddd, $J 16,8,2), 5.44$ $(1 \mathrm{H}, \mathrm{dq}, J 16,7), 6.83(2 \mathrm{H}, \mathrm{d}, J 8.5), 6.87(1 \mathrm{H}, \mathrm{dd}, J 8.5,8.5)$ and $7.26(2 \mathrm{H}$, ddd, $J 8.5,8.5,1) ; \delta_{\mathrm{C}}\left(\mathrm{CDCl}_{3}\right) 17.8(\mathrm{q}), 23.8(\mathrm{t}), 29.2$ (t), $33.6(\mathrm{t}), 34.3(\mathrm{q}), 46.9(\mathrm{~d}, 2 \mathrm{C}), 58.2(\mathrm{~d}), 116.4(\mathrm{~d}, 2 \mathrm{C}), 117.3$ (s), 120.5 (d), 125.4 (d), $129.3(\mathrm{~d}, 2$ C), 133.9 (d) and $149.6(\mathrm{~s})$; $m /=254\left(12 \%, \mathrm{M}^{+}\right)$and $145(100)$.

\section{trans-2-( $N$-Methylanilino)-2-(5-prop-1-enylcyclopent-2-enyl)- acetonitrile 18}

The free-radical cyclisation of 6 [a mixture of $2 E$ - and $2 Z$ isomers (46:54), $165 \mathrm{mg}$ ], by a procedure similar to that for 17 , gave $18 \mathrm{a}(32 \mathrm{mg}, 25 \%$ ) and $18 \mathrm{~b}(55 \mathrm{mg}, 44 \%)$. 18a: Yellow oil; TLC [EtOAc-hexane $(2: 98)] R_{\mathrm{F}} 0.09 ; v_{\text {max }}($ neat $) / \mathrm{cm}^{-1} 2222$ $(\mathrm{CN}) ; \delta_{\mathrm{H}}\left(\mathrm{CDCl}_{3}\right) 1.64(3 \mathrm{H}, \mathrm{d}, J 5), 2.15-2.23(1 \mathrm{H}, \mathrm{m}), 2.56-$ $2.70(1 \mathrm{H}, \mathrm{m}), 2.91(3 \mathrm{H}, \mathrm{s}), 2.98-3.08(2 \mathrm{H}, \mathrm{m}), 4.22(1 \mathrm{H}, \mathrm{d}, J 9$, 2-H), 5.38-5.44 (2 H, m), 5.75-5.83(1 H, m), 5.91-5.97 (1 H, m), 6.86-7.01 $(3 \mathrm{H}, \mathrm{m})$ and 7.25-7.34 $(2 \mathrm{H}, \mathrm{m}) ; \delta_{\mathrm{C}}\left(\mathrm{CDCl}_{3}\right) 17.8(\mathrm{q})$, 34.8 (q), 38.9 (t), 44.4 (d), 54.0 (d), 58.0 (d, C-2), 116.1 (d, 2 C), $116.3(\mathrm{~s}, \mathrm{CN}), 120.4$ (d), 125.1 (d), 128.8 (d), 129.4 (d, 2 C), 134.0 (d), $134.1(\mathrm{~d})$ and $149.4(\mathrm{~s}) ; m / z 252\left(8 \%, \mathrm{M}^{+}\right)$and $145(100)$. 18b: Yellow oil; TLC [EtOAc-hexane $(2: 98)$ ] $R_{\mathrm{F}} 0.07$; $v_{\max }($ neat $) / \mathrm{cm}^{-1} 2232(\mathrm{CN}) ; \delta_{\mathrm{H}}\left(\mathrm{CDCl}_{3}\right) 1.68(3 \mathrm{H}, \mathrm{dd}, J 5,1)$, $2.20-2.31(1 \mathrm{H}, \mathrm{m}), 2.65-2.78(1 \mathrm{H}, \mathrm{m}), 2.92(3 \mathrm{H}, \mathrm{s}), 2.99-3.12$ $(2 \mathrm{H}, \mathrm{m}), 4.26(1 \mathrm{H}, \mathrm{d}, J 9,2-\mathrm{H}), 5.43-5.63(2 \mathrm{H}, \mathrm{m}), 5.65-5.77$ $(1 \mathrm{H}, \mathrm{m}), 5.81-5.88(1 \mathrm{H}, \mathrm{m}), 6.89(2 \mathrm{H}, \mathrm{d}, J 8), 6.91(1 \mathrm{H}$, ddd, $J 8$, $8,1)$ and $7.28(2 \mathrm{H}$, ddd, $J 8,8,1) ; \delta_{\mathrm{C}}\left(\mathrm{CDCl}_{3}\right) 17.8(\mathrm{q}), 34.7(\mathrm{q})$, $39.5(\mathrm{t}), 44.0(\mathrm{~d}), 54.0(\mathrm{~d}), 58.3(\mathrm{~d}), 116.5(\mathrm{~d}, 2 \mathrm{C}), 117.1(\mathrm{~s}), 120.7$ (d), 125.8 (d), 129.2 (d), 129.5 (d, 2 C), 132.7 (d), 133.6 (d) and $149.6(\mathrm{~s}) ; m / z 252\left(11 \%, \mathrm{M}^{+}\right), 145(100)$ (Found: $\mathrm{M}^{+}, 252.1630$. Calc. for $\mathrm{C}_{17} \mathrm{H}_{20} \mathrm{~N}_{2}: M, 252.1626$ ).

\section{2-(N-Methylanilino)-2-(5-methyl-2-prop-1-enylcyclopentyl)- acetonitrile 19}

The free-radical cyclisation of 7 [a mixture of $2 E$ - and $2 Z$ isomers $(42: 58), 174 \mathrm{mg}$ ], by a procedure similar to that for 17, gave a mixture of 19a and 19b (79 mg, 59\%; 55:45), 19c (29 mg, 22\%) and 19d ( $8 \mathrm{mg}, 6 \%$ ). 19a/19b: Yellow oil; TLC [EtOAc-hexane $(2: 98)] R_{\mathrm{F}} 0.10 ; v_{\max }($ neat $) / \mathrm{cm}^{-1} 2225(\mathrm{CN})$; $\delta_{\mathrm{H}}\left(\mathrm{CDCl}_{3}\right) 1.05(3 \mathrm{H}, \mathrm{d}, J 7, \mathrm{Me}) / 1.15(\mathrm{~d}, J 4.5), 1.20-1.56$ $(2 \mathrm{H}, \mathrm{m}), 1.43(\mathrm{~d}, J 5, \mathrm{Me}) / 1.33(\mathrm{~d}, J 5.4), 1.41-2.10(4 \mathrm{H}, \mathrm{m})$, 2.30-2.52(1 H, m)/2.18-2.31 (1 H, m), $2.82(3 \mathrm{H}, \mathrm{s}, \mathrm{NMe}) / 2.86$ $(\mathrm{s}), 4.39(1 \mathrm{H}, \mathrm{d}, J 11,2-\mathrm{H}) / 4.30(\mathrm{~d}, J 9), 5.32-5.52(2 \mathrm{H}, \mathrm{m}) / 5.13-$ 5.34 $(2 \mathrm{H}, \mathrm{m}), 6.83-6.91(3 \mathrm{H}, \mathrm{m})$ and 7.24-7.30 $(2 \mathrm{H}, \mathrm{m})$; $\delta_{\mathrm{C}}\left(\mathrm{CDCl}_{3}\right) 15.2(\mathrm{q}), 17.8 / 22.0(\mathrm{q}), 32.0 / 31.0(\mathrm{t}), 32.7 / 33.7(\mathrm{t})$, $34.8 / 33.9$ (q, NMe), 36.0/37.6 (d), 47.9/44.3 (d), 55.1/49.7 (d), $58.3 / 54.2(\mathrm{~d}, \mathrm{C}-2), 115.8 / 115.7$ (d, $\left.2 \mathrm{C}, \mathrm{C}-2^{\prime}\right), 117.7 / 116.2(\mathrm{~s}, \mathrm{CN})$, $120.1 / 119.9$ (d), $124.7 / 123.7$ (d), 129.3/129.2 (d, 2 C, C-3'), $134.6 / 134.8(\mathrm{~d})$ and $149.5 / 149.7\left(\mathrm{~s}, \mathrm{C}-1^{\prime}\right) ; m / z 268\left(6 \%, \mathrm{M}^{+}\right), 145$ (84) and $107(100)$. 19c: Yellow oil; TLC [EtOAc-hexane $(2: 98)] R_{\mathrm{F}} 0.09 ; v_{\max }($ neat $) / \mathrm{cm}^{-1} 2225(\mathrm{CN}) ; \delta_{\mathrm{H}}\left(\mathrm{CDCl}_{3}\right) 1.06(3$ $\mathrm{H}, \mathrm{d}, J 6.6), 1.31-1.39(1 \mathrm{H}, \mathrm{m}), 1.44-1.93(4 \mathrm{H}, \mathrm{m}), 1.64(3 \mathrm{H}, \mathrm{d}$, J 7), 1.93-2.03 (1 H, m), 2.40-2.47 (1 H, m), $2.88(3 \mathrm{H}, \mathrm{s}), 4.27$ $(1 \mathrm{H}, \mathrm{d}, J 10,2-\mathrm{H}), 5.35-5.56(2 \mathrm{H}, \mathrm{m}), 6.88-6.92(3 \mathrm{H}, \mathrm{m})$ and $7.28(2 \mathrm{H}, \mathrm{dd}, J 8,8,1) ; \delta_{\mathrm{C}}\left(\mathrm{CDCl}_{3}\right) 17.9(\mathrm{q}), 21.1(\mathrm{q}), 32.3(\mathrm{t})$, $33.4(\mathrm{t}), 34.9(\mathrm{q}), 38.5(\mathrm{~d}), 47.0(\mathrm{~d}), 53.6(\mathrm{~d}), 58.8(\mathrm{~d}), 116.3(\mathrm{~d}, 2$ C), 117.2 (s), 120.5 (d), 125.0 (d), 129.3 (d, 2 C), 134.6 (d) and $149.6(\mathrm{~s}) ; m / 2268\left(7 \%, \mathrm{M}^{+}\right), 145(100)$ and $107(90)$ (Found: $\mathrm{M}^{+}$, 268.1934. Calc. for $\left.\mathrm{C}_{18} \mathrm{H}_{24} \mathrm{~N}_{2}: M, 268.1939\right)$. 19d: Yellow oil; TLC [EtOAc-hexane $(2: 98)] R_{\mathrm{F}} 0.07 ; v_{\max }$ (neat) $/ \mathrm{cm}^{-1} 2225$ 
$(\mathrm{CN}) ; \delta_{\mathrm{H}}\left(\mathrm{CDCl}_{3}\right) 0.83(3 \mathrm{H}, \mathrm{d}, J 7), 1.42-1.53(1 \mathrm{H}, \mathrm{m}), 1.68(3$ $\mathrm{H}$, dd, $J 6,1), 1.75-1.86(1 \mathrm{H}, \mathrm{m}), 1.95-2.07(1 \mathrm{H}, \mathrm{m}), 2.10-2.19$ $(1 \mathrm{H}, \mathrm{m}), 2.22-2.26(1 \mathrm{H}, \mathrm{m}), 2.44-2.49(1 \mathrm{H}, \mathrm{m}), 2.89(3 \mathrm{H}, \mathrm{s})$, 3.71-3.75 (1 H, m), 4.25 (1 H, J11, 2-H), $5.47(1 \mathrm{H}, \mathrm{dd}, J 17,8)$, $5.48(1 \mathrm{H}, \mathrm{dq}, J 17,6), 6.90(2 \mathrm{H}, \mathrm{dd}, J 8), 6.94(1 \mathrm{H}, \mathrm{dd}, J 8,8)$ and $7.28(2 \mathrm{H}$, dd, $J 8,8) ; \delta_{\mathrm{C}}\left(\mathrm{CDCl}_{3}\right) 15.2(\mathrm{q}), 17.8(\mathrm{q}), 31.4(\mathrm{t})$, $31.7(\mathrm{t}), 34.9$ (q), 35.6 (d), 44.5 (d), 49.4 (d), 54.4 (d), 116.4 (d, 2 C), 117.7 (s), 120.6 (d), 126.0 (d), 129.3 (d, 2 C), 134.3 (d) and $149.2(\mathrm{~s}) ; \mathrm{m} / \mathrm{z} 268\left(13 \%, \mathrm{M}^{+}\right), 145(100)$ and $107(53)$.

\section{trans-2-( $N$-Methylanilino)-2-(3-prop-1-enylbicyclo[3.3.0] oct- 1(5)-en-2-yl)acetonitrile 20}

The free-radical cyclisation of 8 [a mixture of $(2 E)$ - and (2Z)isomers $(20: 80), 144 \mathrm{mg}$ ], by a procedure similar to that for 17 , gave 20a (48 mg, 33\%) and 20b (72 mg, 49\%). 20a: Yellow oil; TLC [EtOAc-hexane $(2: 98)] \boldsymbol{R}_{\mathrm{F}} 0.10 ; v_{\max }($ neat $) / \mathrm{cm}^{-1} 2223$ $(\mathrm{CN}) ; \delta_{\mathrm{H}}\left(\mathrm{CDCl}_{3}\right) 1.52-1.72(2 \mathrm{H}, \mathrm{m}), 1.63(3 \mathrm{H}, \mathrm{d}, J 6), 1.90$ $2.03(2 \mathrm{H}, \mathrm{m}), 2.12-2.32(4 \mathrm{H}, \mathrm{m}), 2.86(3 \mathrm{H}, \mathrm{s}), 2.85-2.93(1 \mathrm{H}$, $\mathrm{m}), 3.02-3.12(1 \mathrm{H}, \mathrm{m}), 4.22(1 \mathrm{H}, \mathrm{d}, J 10,2-\mathrm{H}), 5.34(1 \mathrm{H}, \mathrm{dd}, J$ $16,6), 5.50(1 \mathrm{H}, \mathrm{dq}, J 16,6), 6.86-6.96(3 \mathrm{H}, \mathrm{m})$ and $7.25-7.32$ $(2 \mathrm{H}, \mathrm{m}) ; \delta_{\mathrm{C}}\left(\mathrm{CDCl}_{3}\right) 17.7(\mathrm{q}), 26.8(\mathrm{t}), 27.8(\mathrm{t}), 29.2(\mathrm{t}), 34.5(\mathrm{q})$, $35.4(\mathrm{t}), 50.7$ (d), 51.0 (d), $57.4(\mathrm{~d}, \mathrm{C}-2), 116.1(\mathrm{~d}, 2 \mathrm{C}), 118.0(\mathrm{~s})$, 120.3 (d), 126.2 (d), 129.2 (d, 2 C), 134.8 (d), 142.4 (s), 146.2 (s) and $148.9\left(\mathrm{~s}, \mathrm{C}-1^{\circ}\right) ; \mathrm{m} / z 292\left(8 \%, \mathrm{M}^{+}\right)$and $147(100)$. 20b: Yellow oil; TLC [EtOAc-hexane $(2: 98)] R_{\mathrm{F}} 0.08 ; v_{\max }($ neat $) / \mathrm{cm}^{-1}$ $2231(\mathrm{CN}) ; \delta_{\mathrm{H}}\left(\mathrm{CDCl}_{3}\right) 1.52-1.62(2 \mathrm{H}, \mathrm{m}), 1.67(3 \mathrm{H}, \mathrm{d}, J 6)$, 1.92-2.31 $(5 \mathrm{H}, \mathrm{m}), 2.48-2.63(1 \mathrm{H}, \mathrm{m}), 2.81-2.93(1 \mathrm{H}, \mathrm{m}), 2.91$ (3 H, s), 3.10-3.18 (1 H, m), $4.33(1 \mathrm{H}, \mathrm{d}, J 9,2-\mathrm{H}), 5.42-5.58$ $(2 \mathrm{H}, \mathrm{m}), 6.85-6.89(3 \mathrm{H}, \mathrm{m})$ and $7.24-7.32(2 \mathrm{H}, \mathrm{m})$; $\delta_{\mathrm{C}}\left(\mathrm{CDCl}_{3}\right) 17.9(\mathrm{q}), 26.8(\mathrm{t}), 27.9(\mathrm{t}), 29.2(\mathrm{t}), 34.6(\mathrm{q}), 35.9(\mathrm{t})$, 50.0 (d), 51.0 (d), $57.8(\mathrm{~d}, \mathrm{C}-2), 116.3(\mathrm{~d}, 2 \mathrm{C}), 117.2(\mathrm{~s}), 120.4(\mathrm{~d})$, 125.1 (d), 129.3 (d, 2 C), 134.4 (d), 144.0 (s), 148.2 (s) and 149.6 (s); $m / z$ (rel. intensity) $292\left(13 \%, \mathrm{M}^{+}\right.$) and 147 (100) (Found: $\mathrm{M}^{+}, 292.1935$. Calc. for $\left.\mathrm{C}_{20} \mathrm{H}_{24} \mathrm{~N}_{2}: M, 292.1939\right)$.

trans-2-(2,3-Dihydro-2-prop-1-enyl-1 $H$-indan-1-yl)-2- $(N$ methylanilino)acetonitrile 21 and trans-2-(2,3-dihydro-2-prop1-enyl-1 $H$-indan-1-yl)-3,3-dimethyl-2-( $N$-methylanilino)butane-1,4-dinitrile 22

The free-radical cyclisation of 9 [a mixture of $(2 E)$ - and (2Z)isomers (26:74), $305 \mathrm{mg}$ ], by a procedure similar to that for 17 except that 1.2 equiv. of AIBN was used, gave $21 \mathrm{a}(49 \mathrm{mg}, 21 \%$ ), $21 \mathrm{~b}$ (43 mg, 18\%), 22a (107 mg, 37\%) and 22b (69 mg, 23\%). 21a: Yellow oil; TLC [EtOAc-hexane $(5: 95)] \boldsymbol{R}_{\mathrm{F}} 0.24 ; v_{\max }$ (neat)/ $\mathrm{cm}^{-1} 2225(\mathrm{CN}) ; \delta_{\mathrm{H}}\left(\mathrm{CDCl}_{3}\right) 1.60(3 \mathrm{H}, \mathrm{d}, J 4.5), 2.71(1 \mathrm{H}, \mathrm{dd}$, $J 16,1.5,3-\mathrm{H}), 2.99(3 \mathrm{H}, \mathrm{s}), 3.08-3.15(1 \mathrm{H}, \mathrm{m}, 2-\mathrm{H}), 3.21(1 \mathrm{H}$, dd, $J 16,7.5,3-\mathrm{H}), 3.45(1 \mathrm{H}, \mathrm{dd}, J 11,2,1-\mathrm{H}), 4.24(1 \mathrm{H}, \mathrm{d}, J 11)$, 5.42-5.49 (2 H, m), 6.88-6.95 $(3 \mathrm{H}, \mathrm{m}), 7.22-7.32(5 \mathrm{H}, \mathrm{m})$ and 7.59-7.63 (1 H, m); $\delta_{\mathrm{C}}\left(\mathrm{CDCl}_{3}\right) 17.8(\mathrm{q}), 34.5(\mathrm{q}), 37.2(\mathrm{t}), 43.8$ (d), 53.0 (d), 57.9 (d), 116.3 (d, 2 C), $117.5(\mathrm{~s}, \mathrm{CN}), 120.7$ (d), 125.0 (d), 125.2 (d), 126.1 (d), 126.8 (d), 128.3 (d), 129.4 (d, 2 C), 133.8 (d), 140.0 (s), 142.8 (s) and $149.4(\mathrm{~s}) ; \mathrm{m} / \mathrm{z} 302\left(7 \%, \mathrm{M}^{+}\right)$, 157 (30), 145 (100). 21b: White solid, $\mathrm{mp} 69-71^{\circ} \mathrm{C}$; TLC [EtOAc-hexane $(5: 95)] \boldsymbol{R}_{\mathrm{F}} 0.21 ; v_{\max }(\mathrm{KBr}) / \mathrm{cm}^{-1} 2225(\mathrm{CN})$; $\delta_{\mathrm{H}}\left(\mathrm{CDCl}_{3}\right) 1.67(3 \mathrm{H}, \mathrm{d}, J 5), 2.80(1 \mathrm{H}, \mathrm{dd}, J 16,4.5,3-\mathrm{H}), 2.98$ (3 H, s), 3.00-3.07 (1 H, m, 2-H), 3.28(1 H, dd, J 16, 8, 3-H), 3.46 $(1 \mathrm{H}, \mathrm{dd}, J 10,4.5,1-\mathrm{H}), 4.42(1 \mathrm{H}, \mathrm{d}, J 10), 5.52-5.61(2 \mathrm{H}, \mathrm{m})$, $6.73(2 \mathrm{H}, \mathrm{d}, J 8), 6.87(1 \mathrm{H}$, ddd, $J 8,8,1)$ and $7.11-7.39(6 \mathrm{H}, \mathrm{m})$; $\delta_{\mathrm{C}}\left(\mathrm{CDCl}_{3}\right) 17.8(\mathrm{q}), 35.0(\mathrm{q}), 38.2(\mathrm{t}), 45.5(\mathrm{~d}), 51.8(\mathrm{~d}), 58.9(\mathrm{~d})$, 116.8 (s), 116.9 (d, 2 C), 120.9 (d), 124.7 (d), 126.1 (d), 126.7 (d), 128.0 (d), 129.1 (d), 129.2 (d, 2 C), 133.2 (d), 141.1 (s), 142.4 (s) and $149.6(\mathrm{~s}) ; \mathrm{m} / z$ 302 $\left(4 \%, \mathrm{M}^{+}\right), 157(24), 146(64), 145(100)$ and 129 (80) (Found: $\mathrm{M}^{+}, 302.1781$. Calc. for $\mathrm{C}_{21} \mathrm{H}_{22} \mathrm{~N}_{2}: M$, 302.1783). The crystal data for $21 \mathbf{b}$ are reported.

22a: White solid, $\mathrm{mp} 110-112^{\circ} \mathrm{C}$; TLC [EtOAc-hexane $(5: 95)] \quad R_{\mathrm{F}} \quad 0.15 ; \quad v_{\max }(\mathrm{KBr}) / \mathrm{cm}^{-1} \quad 2230 \quad(\mathrm{CN}) ; \delta_{\mathrm{H}}\left(\mathrm{CDCl}_{3}\right)$ $1.52(3 \mathrm{H}, \mathrm{s}), 1.58(3 \mathrm{H}, \mathrm{dd}, J 6,1), 1.68(3 \mathrm{H}, \mathrm{s}), 2.45(1 \mathrm{H}, \mathrm{d}, J 17$, $\left.3^{\prime}-\mathrm{H}\right), 3.24(3 \mathrm{H}, \mathrm{s}), 3.26\left(1 \mathrm{H}, \mathrm{dd}, J 8,8,2^{\prime}-\mathrm{H}\right), 3.67(1 \mathrm{H}, \mathrm{dd}, J 17$,
$\left.8,3^{\prime}-\mathrm{H}\right), 4.24\left(1 \mathrm{H}, \mathrm{s}, 1^{\prime}-\mathrm{H}\right), 5.24(1 \mathrm{H}$, ddd, $J 15,8,1), 5.48(1 \mathrm{H}$, $\mathrm{dq}, J 15,6), 7.04(1 \mathrm{H}, \mathrm{dd}, J 8,8), 7.23-7.33(7 \mathrm{H}, \mathrm{m})$ and 7.85 $(1 \mathrm{H}, \mathrm{d}, J 8) ; \delta_{\mathrm{C}}\left(\mathrm{CDCl}_{3}\right) 17.7(\mathrm{q}), 27.6(\mathrm{q}), 27.8(\mathrm{q}), 37.7(\mathrm{q})$, $38.6(\mathrm{t}), 40.4(\mathrm{~s}), 44.7(\mathrm{~d}), 59.3(\mathrm{~d}), 71.6(\mathrm{~s}), 116.0(\mathrm{~s}, \mathrm{CN}), 119.7(\mathrm{~s}$, $\mathrm{CN}), 123.0(\mathrm{~d}), 123.1$ (d, 2C), 124.6(d), 125.7 (d), 125.9 (d), 128.5 (d, 2 C), 128.7 (d), 134.1 (d), $138.4(\mathrm{~s}), 145.9(\mathrm{~s})$ and $149.1(\mathrm{~s}) ; \mathrm{m} / \mathrm{z}$ $369\left(2 \%, \mathrm{M}^{+}\right)$and $212(100)$ (Found: $\mathrm{M}^{+}, 369.2208$. Calc. for $\mathrm{C}_{25} \mathrm{H}_{27} \mathrm{~N}_{3}: M, 369.2205$ ). 22b: White solid, mp 146-148 ${ }^{\circ} \mathrm{C}$; TLC [EtOAc-hexane $(5: 95)] R_{\mathrm{F}} 0.10 ; v_{\max }(\mathrm{KBr}) / \mathrm{cm}^{-1} 2250$ and $2230 ; \delta_{\mathrm{H}}\left(\mathrm{CDCl}_{3}\right) 1.63(3 \mathrm{H}, \mathrm{s}), 1.65(3 \mathrm{H}, \mathrm{d}, J 7), 1.84$ $(3 \mathrm{H}), 2.76(1 \mathrm{H}, \mathrm{d}, J 16.6), 3.28(3 \mathrm{H}, \mathrm{s}), 3.59(1 \mathrm{H}, \mathrm{t}, J 8), 3.81$ $(1 \mathrm{H}, \mathrm{dd}, J 16.6,8,3-\mathrm{H}), 4.01(1 \mathrm{H}, \mathrm{s}), 5.56(1 \mathrm{H}, \mathrm{dq}, J 14,7), 5.57$ $(1 \mathrm{H}, \mathrm{dd}, J 14,8), 6.61(2 \mathrm{H}, \mathrm{dd}, J 8,1), 6.93(1 \mathrm{H}, \mathrm{ddd}, J 8,1)$, 7.06-7.16 $(3 \mathrm{H}, \mathrm{m})$ and $7.25-7.36(3 \mathrm{H}, \mathrm{m}) ; \delta_{\mathrm{C}}\left(\mathrm{CDCl}_{3}\right) 17.8(\mathrm{q})$, 25.1 (q), 28.1 (q), 38.7 (t), 38.8 (q), 43.5(d), 43.6 (s), 56.9 (d), 72.1 (s), 116.9 (s), 123.4 (s), 123.6 (d), 124.4 (d, 2 C), 124.9 (d), 125.1 (d), 126.5 (d), 127.8 (d), 128.1 (d, 2 C), 128.5 (d), 134.3 (d), 139.8 (s), 144.1 (s) and $149.3(\mathrm{~s}) ; \mathrm{m} / z 369\left(1 \%, \mathrm{M}^{+}\right)$and $212(100)$. The crystal data for $\mathbf{2 2 b}$ are reported.

\section{trans-2-(2,3-Dihydro-2-(2-phenylvinyl-1 H-indan-1-yl)-2- \\ ( $N$-methylanilino)acetonitrile 23}

The free-radical cyclisation of 10 [a mixture of $(2 E, 5 E)$ - and $(2 Z, 5 E)$-isomers $(33: 67), 100 \mathrm{mg}]$, by a procedure similar to that for 17, gave $23 \mathrm{a}(41 \mathrm{mg}, 52 \%)$ and $23 \mathrm{~b}(24 \mathrm{mg}, 30 \%)$. 23a: Yellow oil; TLC [EtOAc-hexane $(2: 98)] R_{\mathrm{F}} 0.13 ; v_{\max }($ neat $) / \mathrm{cm}^{-1}$ 2225; $\delta_{\mathrm{H}}\left(\mathrm{CDCl}_{3}\right) 2.82-2.96(1 \mathrm{H}, \mathrm{m}), 3.05(3 \mathrm{H}, \mathrm{s}), 3.28-3.44$ $(2 \mathrm{H}, \mathrm{m}), 3.63(1 \mathrm{H}, \mathrm{dd}, J 11,1.5), 4.37(1 \mathrm{H}, \mathrm{d}, J 11), 6.25$ $(1 \mathrm{H}, \mathrm{dd}, J 16,8), 6.45(1 \mathrm{H}, \mathrm{d}, J 16), 6.46(1 \mathrm{H}, \mathrm{dd}, J 8), 1)$, 6.96-7.06 $(2 \mathrm{H}, \mathrm{m}), 7.20-7.41(10 \mathrm{H}, \mathrm{m})$ and 7.68-7.72 $(1 \mathrm{H}$, $\mathrm{m}) ; \delta_{\mathrm{C}}\left(\mathrm{CDCl}_{3}\right) 34.7(\mathrm{q}), 37.3(\mathrm{t}), 44.6(\mathrm{~d}), 52.9(\mathrm{~d}), 58.0(\mathrm{~d}), 116.5$ (d, 2 C), 117.4 (s), 119.6 (d), 120.9 (d), 125.3 (d), 126.1 (d, 2 C), 127.0 (d), 127.4 (d), 128.5 (d, 2 C), 129.5 (d, 2 C), 129.9 (d), 132.5 (d), 137.0 (s), 139.8 (s), $142.6(\mathrm{~s})$ and $149.4(\mathrm{~s}) ; m / z 364(22 \%$, $\mathrm{M}^{+}$), 145 (74) and 91 (100) (Found: $\mathrm{M}^{+}, 364.1917$. Calc. for $\left.\mathrm{C}_{26} \mathrm{H}_{24} \mathrm{~N}_{2}: M, 364.1939\right)$. 23b: Yellow oil; TLC [EtOAc-hexane $(2: 98)] R_{\mathrm{F}} 0.10 ; v_{\max }$ (neat) $/ \mathrm{cm}^{-1} 2234 ; \delta_{\mathrm{H}}\left(\mathrm{CDCl}_{3}\right) 2.82-2.92$ $(1 \mathrm{H}, \mathrm{m}), 3.00(3 \mathrm{H}, \mathrm{s}), 3.22-3.35(1 \mathrm{H}, \mathrm{m}), 3.41(1 \mathrm{H}, \mathrm{dd}, J 16,8)$, $3.59(1 \mathrm{H}, \mathrm{dd}, J 10,4), 4.49(1 \mathrm{H}, \mathrm{d}, J 10), 6.28(1 \mathrm{H}, \mathrm{dd}, J 16,8)$, $6.56(1 \mathrm{H}, \mathrm{d}, J 16), 6.74(2 \mathrm{H}, \mathrm{d}, J 8), 6.94(1 \mathrm{H}, \mathrm{dd}, J 8,8)$ and 7.12--7.41 (11 H, m); $\delta_{\mathrm{C}}\left(\mathrm{CDCl}_{3}\right) 35.2(\mathrm{q}), 38.1(\mathrm{t}), 45.7(\mathrm{~d}), 52.2$ (d), 58.8 (d), 114.7 (s), 116.9 (d, 2 C), 121.1 (d), 124.5 (d), 124.9 (d), 126.2, (d, 2 C), 126.9 (d), 127.4 (d), 128.2 (d), 128.5 (d, 2 C), 129.3 (d, 2 C), 130.7 (d), 132.0 (d), 137.0 (s), 140.8 (s), $140.9(\mathrm{~s})$ and $149.6(\mathrm{~s}) ; m / z 364\left(8 \%, \mathrm{M}^{+}\right), 219(43), 145(86)$ and $91(100)$.

\section{trans-2-[2-(2-Benzyloxyvinyl)cyclopentyl]-2-( $N$-methylanilino)-} acetonitrile 24

The free-radical cyclisation of 11 [(2Z,5Z)-configuration, 180 $\mathrm{mg}, \mathrm{X}=\mathrm{Br}$ or $\mathrm{I}(1: 1)]$, by a procedure similar to that for 17 , gave $24 \mathrm{a}(20 \mathrm{mg}, 16 \%), 24 \mathrm{~b}(77 \mathrm{mg}, 56 \%)$ and $24 \mathrm{c}(12 \mathrm{mg}, 9 \%)$. 24a (Z-configuration): Yellow oil; TLC [EtOAc-hexane (5:95)] $R_{\mathrm{F}} \quad 0.14 ; \quad v_{\max }($ neat $) / \mathrm{cm}^{-1} 2221 ; \delta_{\mathrm{H}}\left(\mathrm{CDCl}_{3}\right) \quad 1.22$ $1.40(3 \mathrm{H}, \mathrm{m}), 1.69-2.13(4 \mathrm{H}, \mathrm{m}), 2.88(3 \mathrm{H}, \mathrm{s}), 2.87-2.95(1 \mathrm{H}$, $\mathrm{m}), 4.32(1 \mathrm{H}, \mathrm{dd}, J 9.5,6), 4.56(1 \mathrm{H}, \mathrm{d}, J 6), 4.68(1 \mathrm{H}, \mathrm{d}, J 12)$ $4.77(1 \mathrm{H}, \mathrm{d}, J 12), 6.09(1 \mathrm{H}, \mathrm{dd}, J 6,1), 6.79(2 \mathrm{H}, \mathrm{dd}, J 8,1), 6.86$ $(1 \mathrm{H}$, ddd, $J 8,8,1)$ and 7.18-7.31 $(7 \mathrm{H}, \mathrm{m}) ; \delta_{\mathrm{C}}\left(\mathrm{CDCl}_{3}\right) 24.1(\mathrm{t})$, $29.4(\mathrm{t}), 32.8(\mathrm{t}), 35.1(\mathrm{q}), 39.2(\mathrm{~d}), 48.5(\mathrm{~d}), 57.4(\mathrm{~d}), 73.9(\mathrm{t}), 110.2$ (d), $115.6(\mathrm{~d}, 2 \mathrm{C}), 117.4(\mathrm{~s}, \mathrm{CN}), 119.8(\mathrm{~d}), 127.5(\mathrm{~d}, 2 \mathrm{C}), 128.0$ (d), $128.5(\mathrm{~d}, 2 \mathrm{C}), 129.3(\mathrm{~d}, 2 \mathrm{C}), 137.3(\mathrm{~s}), 145.3(\mathrm{~d})$ and $149.6(\mathrm{~s})$; $m / z 346\left(4 \%, \mathrm{M}^{+}\right), 145(49), 107(70)$ and 91 (100) (Found: $\mathrm{M}^{+}$, 346.2036. Calc. for $\left.\mathrm{C}_{23} \mathrm{H}_{26} \mathrm{~N}_{2} \mathrm{O}: M, 346.2045\right)$. 24b ( $Z$ configuration): Yellow oil; TLC [EtOAc-hexane (5:95)] $R_{\mathrm{F}}$ $0.12 ; v_{\max }($ neat $) / \mathrm{cm}^{-1} 2224 ; \delta_{\mathrm{H}}\left(\mathrm{CDCl}_{3}\right) 1.39-1.69(4 \mathrm{H}, \mathrm{m})$, 1.82-2.01 (2 H, m), 2.16-2.29 (1 H, m), $2.85(3 \mathrm{H}, \mathrm{s}), 2.80-2.92$ $(1 \mathrm{H}, \mathrm{m}), 2.19(1 \mathrm{H}, \mathrm{d}, J 10), 4.45(1 \mathrm{H}, \mathrm{dd}, J 9,6), 4.77(1 \mathrm{H}, \mathrm{d}, J$ 12), $4.87(1 \mathrm{H}, \mathrm{d}, J 12), 6.11(1 \mathrm{H}, \mathrm{d}, J 6), 6.88(2 \mathrm{H}, \mathrm{d}, J 8), 6.90$ 
$(1 \mathrm{H}$, ddd, $J 8,8,1)$ and $7.22-7.37(7 \mathrm{H}, \mathrm{m}) ; \delta_{\mathrm{C}}\left(\mathrm{CDCl}_{3}\right) 23.3(\mathrm{t})$, $29.3(\mathrm{t}), 34.2(\mathrm{t}), 34.3(\mathrm{q}), 39.7(\mathrm{~d}), 47.6(\mathrm{~d}), 58.1(\mathrm{~d}), 73.8(\mathrm{t}), 110.3$ (d), 116.3 (d, 2 C), 117.7 (s, CN), 120.4 (d), 127.6 (d, 2 C), 127.9 (d), $128.5(\mathrm{~d}, 2 \mathrm{C}), 129.3(\mathrm{~d}, 2 \mathrm{C}), 138.8(\mathrm{~s}), 144.4(\mathrm{~d})$ and $145.4(\mathrm{~s})$; $m / z 346\left(4 \%, \mathrm{M}^{+}\right), 145(56), 107(72)$ and $91(100) .24 \mathrm{c}(E-$ configuration): Yellow oil; TLC [EtOAc-hexane $(5: 95)] R_{\mathrm{F}}$ $0.08 ; v_{\max }($ neat $) / \mathrm{cm}^{-1} 2205 ; \delta_{\mathrm{H}}\left(\mathrm{CDCl}_{3}\right) 1.45-1.70(4 \mathrm{H}, \mathrm{m})$, 1.78-2.00 (2 H, m), 2.09-2.40 (2 H, m), $2.87(3 \mathrm{H}, \mathrm{s}), 4.15(1$ $\mathrm{H}, \mathrm{d}, J 10), 4.75(2 \mathrm{H}, \mathrm{s}), 4.92(1 \mathrm{H}, \mathrm{dd}, J 13,9), 6.45(1 \mathrm{H}, \mathrm{d}$, $J 13), 6.88-6.95(3 \mathrm{H}, \mathrm{m})$ and $7.19-7.36(7 \mathrm{H}, \mathrm{m}) ; \delta_{\mathrm{C}}\left(\mathrm{CDCl}_{3}\right)$ 23.7 (t), 29.3 (t), 34.5 (q), 34.7 (t), 42.9 (d), 47.7 (d), 58.1 (d), $71.4(\mathrm{t}), 108.2(\mathrm{~d}), 113.5(\mathrm{~s}, \mathrm{CN}), 116.5(\mathrm{~d}, 2 \mathrm{C}), 120.6(\mathrm{~d})$, 127.6 (d, 2 C), 127.8 (d), 128.4 (d, 2 C), 129.3 (d, 2 C), 137.1 (s), 146.5 (d) and $149.6(\mathrm{~s}) ; m / z 346\left(8, \mathrm{M}^{+}\right), 145(50), 107$ (52) and $91(100)$.

\section{trans-2-(2,3-Dihydro-2-(2-phenylsulfanylvinyl)-1 $H$-indan-1-yl)- 2-( $N$-methylanilino)acetonitrile 25}

The free-radical cyclisation of 12 (2E,5E-configuration, 150 $\mathrm{mg}$ ), by a procedure similar to that for 17 , gave $25 \mathrm{a}(61 \mathrm{mg}$, $48 \%$ ) and $25 \mathrm{~b}(52 \mathrm{mg}, 41 \%) .25 \mathrm{a}$ : White solid, $\mathrm{mp} 134-136^{\circ} \mathrm{C}$; TLC [EtOAc-hexane) $2: 98)] R_{\mathrm{F}} 0.13 ; v_{\max }(\mathrm{KBr}) / \mathrm{cm}^{-1} 2234$; $\delta_{\mathrm{H}}\left(\mathrm{CDCl}_{3}\right) 2.82(1 \mathrm{H}, \mathrm{dd}, J 19,6), 3.00(3 \mathrm{H}, \mathrm{s}), 3.21-3.30(2 \mathrm{H}$, $\mathrm{m}), 3.55(1 \mathrm{H}, \mathrm{dd}, J 11,2), 4.29(1 \mathrm{H}, \mathrm{d}, J 11), 5.93(1 \mathrm{H}, \mathrm{dd}, J 15$, $8), 6.19(1 \mathrm{H}, \mathrm{d}, J 15), 6.92(2 \mathrm{H}, \mathrm{d}, J 8), 6.93(1 \mathrm{H}, \mathrm{ddd}, J 8,8,1)$, $7.14-7.33(10 \mathrm{H}, \mathrm{m})$ and $7.62-7.66(1 \mathrm{H}, \mathrm{m}) ; \delta_{\mathrm{C}}\left(\mathrm{CDCl}_{3}\right) 34.8$ (q), 37.0 (t), 44.9 (d), 52.5 (d), 58.1 (d), 116.6 (d, 2 C), 117.2 (s), 121.1 (d), 122.6 (d), 125.5 (d), 126.0 (d), 126.4 (d), 127.1 (d), 128.5 (d), 128.8 (d, 2 C), 129.0 (d, 2 C), 129.1 (s), 129.5 (d, 2 C), 137.0 (d), 139.5 (s), 142.2 (s) and $149.3(\mathrm{~s}) ; m / z 396\left(0.1 \%, \mathrm{M}^{+}\right), 251$ (100), 145 (60) and 141 (64) (Found: $\mathrm{M}^{+}, 396.1634$. Calc. for $\left.\mathrm{C}_{26} \mathrm{H}_{24} \mathrm{~N}_{2} \mathrm{~S}: M, 396.1660\right)$. 25b: Yellow oil; TLC [EtOAchexane $(2: 98)] \quad R_{\mathrm{F}} 0.10 ; v_{\max }$ (neat) $/ \mathrm{cm}^{-1} 2215 ; \delta_{\mathrm{H}}\left(\mathrm{CDCl}_{3}\right)$ $2.89(1 \mathrm{H}, \mathrm{dd}, J 14,4), 3.00(3 \mathrm{H}, \mathrm{s}), 3.16-3.29(1 \mathrm{H}, \mathrm{m}), 3.35(1 \mathrm{H}$, dd, $J 15,8), 3.55(1 \mathrm{H}, \mathrm{dd}, J 10,4), 4.47(1 \mathrm{H}, \mathrm{d}, J 10), 6.00(1 \mathrm{H}$, $\mathrm{dd}, J 15,8), 6.36(1 \mathrm{H}, \mathrm{d}, J 15), 6.76(2 \mathrm{H}, \mathrm{d}, J 8), 6.90(1 \mathrm{H}$, ddd, $J$ 8, 8, 1), 7.10-7.36 $(10 \mathrm{H}, \mathrm{m})$ and $7.38(1 \mathrm{H}$, dd, $J 8,8)$; $\delta_{\mathrm{C}}\left(\mathrm{CDCl}_{3}\right) 35.3(\mathrm{q}), 38.1(\mathrm{t}), 45.9(\mathrm{~d}), 51.5(\mathrm{~d}), 59.0(\mathrm{~d}), 116.7$ (s), 117.2 (d, 2 C), 121.3 (d), 123.9 (d), 124.8 (d), 126.1 (d), 128.5 (d), 127.0 (d), 128.2 (d), 129.0 (d, 2 C), 129.1 (d, 2 C), 129.3 (d, $2 \mathrm{C}), 135.5(\mathrm{~s}), 135.9$ (d, $140.7(\mathrm{~s})$ and $148.8(\mathrm{~s}) ; \mathrm{m} /=396(2 \%$, $\left.\mathrm{M}^{+}\right), 251(100), 145(62)$ and $141(60)$.

\section{Methyl trans-2-\{1-[cyano( $N$-methylanilino)methyl]- 2,3-dihydroindan-2-yl\}acrylate 26 and methyl trans-2-\{2- [2-cyano-2-( $N$-methylanilino)vinyl]-2,3-dihydroindan-1-yl $\}$ - acetate 27}

The free-radical cyclisation of 13 (2E,5Z-configuration, 280 $\mathrm{mg}$ ), by a procedure similar to that for 17 , gave an inseparable mixture 26a/26b (45 mg, 6:4; 20\%), 26c ( $90 \mathrm{mg}, 40 \%$ ), 26d (40 $\mathrm{mg}, 17 \%)$ and $27(40 \mathrm{mg}, 17 \%) . \mathbf{2 6 a} / \mathbf{2 6 b}(6: 4,2 E$-configuration): Yellow oil; TLC [EtOAc-hexane $(10: 90)] R_{\mathrm{F}} 0.21 ; v_{\max }$ (neat)/ $\mathrm{cm}^{-1} 2234(\mathrm{CN}), 1719(\mathrm{CO})$ and $1641 ; \delta_{\mathrm{H}}\left(\mathrm{CDCl}_{3}\right) 2.72 / 2.78$ $(1 \mathrm{H}, \mathrm{dd}, J 16,4), 2.93 / 2.90(3 \mathrm{H}, \mathrm{s}, \mathrm{NMe}), 3.38(1 \mathrm{H}, \mathrm{dd}, J 16,8)$, $3.52(1 \mathrm{H}, \mathrm{dd}, J 11,4) / 3.72(1 \mathrm{H}, \mathrm{dd}, J 10.8), 3.56(3 \mathrm{H}, \mathrm{s}, \mathrm{OMe})$, $4.39(1 \mathrm{H}, \mathrm{d}, J 11) / 4.68(1 \mathrm{H}, \mathrm{d}, J 10), 4.39-4.48(1 \mathrm{H}, \mathrm{m})$, $5.75 / 5.86(1 \mathrm{H}, \mathrm{d}, J 11), 6.14 / 6.28(1 \mathrm{H}, \mathrm{dd}, J 11,10), 6.82-6.94$ ( $3 \mathrm{H}, \mathrm{m}), 7.10-7.32(5 \mathrm{H}, \mathrm{m})$ and $7.63-7.68(1 \mathrm{H}, \mathrm{m})$; $\delta_{\mathrm{C}}\left(\mathrm{CDCl}_{3}\right) 34.9 / 35.0(\mathrm{q}), 37.2 / 38.1(\mathrm{t}), 40.9 / 41.7(\mathrm{~d}), 51.1 / 51.3$ (q), 52.6/52.2 (d), 58.0/58.9 (d), 116.6/116.2 (d, 2 C), 117.3/116.8 (s, CN), $118.8 / 120.0$ (d), $120.8 / 121.0$ (d), $125.2 / 124.8$ (d), $125.8 / 126.0$ (d), 127.0 (d), $128.4 / 128.2$ (d), 129.3 (d, 2 C), $139.6 / 140.8$ (s), 142.4/142.2 (s), 149.2/149.3 (s), 150.7/151.2(d) and $166.1 / 166.4(\mathrm{~s}, \mathrm{CO}) ; m / z 346\left(20 \%, \mathrm{M}^{+}\right), 320(50), 201(78)$ and 169 (100) (Found: $\mathrm{M}^{+}, 346.1673$. Calc. for $\mathrm{C}_{22} \mathrm{H}_{22} \mathrm{~N}_{2} \mathrm{O}_{2}$ : $M, 346.1681)$. 26c (2Z-configuration): Yellow oil; TLC [EtOAchexane $(10: 90)] R_{\mathrm{F}} 0.13 ; v_{\max }$ (neat) $/ \mathrm{cm}^{-1} 2224,1721$ (CO) and $1653 ; \delta_{\mathrm{H}}\left(\mathrm{CDCl}_{3}\right) 2.81(1 \mathrm{H}, \mathrm{d}, J 16), 2.96(3 \mathrm{H}, \mathrm{s}), 3.20$
$3.33(1 \mathrm{H}, \mathrm{m}), 3.55(1 \mathrm{H}, \mathrm{dd}, J 11,1), 3.68(3 \mathrm{H}, \mathrm{s}), 3.67-3.75(1 \mathrm{H}$, $\mathrm{m}), 4.37(\mathrm{l} \mathrm{H}, \mathrm{d}, J 11), 5.81(1 \mathrm{H}, \mathrm{d}, 15,5), 6.86-7.02(4 \mathrm{H}, \mathrm{m})$, $7.16-7.33(5 \mathrm{H}, \mathrm{m})$ and $7.58-7.62(1 \mathrm{H}, \mathrm{m}) ; \delta_{\mathrm{C}}\left(\mathrm{CDCl}_{3}\right) 34.7(\mathrm{q})$, 36.0 (t, 43.3 (d), 51.5 (q, OMe), 52.0 (d), 57.9 (d), 116.6 (d, 2 C), 117.0 (s), 120.6(d), 121.2(d), 125.2(d), 126.1 (d), 127.3 (d), 128.7 (d), 129.5 (d, 2 C), 138.9 (s), 141.7 (s), 149.2 (s), 150.1 (d) and $166.7(\mathrm{~s}) ; m / z 347\left(20 \%,[\mathrm{M}+2]^{+}\right), 346\left(40, \mathrm{M}^{+}\right), 320(35)$, $272(30), 201(34), 169(28), 145(100), 141(40), 115(20)$ and 77 (24). 26d (2Z-configuration): Yellow oil; TLC [EtOAc-hexane $(10: 90)] \quad R_{\mathrm{F}} 0.11 ; v_{\max }($ neat $) / \mathrm{cm}^{-1} 2234,1721$ and 1656 ; $\delta_{\mathrm{H}}\left(\mathrm{CDCl}_{3}\right) 2.90(1 \mathrm{H}, \mathrm{dd}, J 16,3), 2.98(3 \mathrm{H}, \mathrm{s}), 3.22-3.30(1 \mathrm{H}$, $\mathrm{m}), 3.39(1 \mathrm{H}, \mathrm{dd}, J 16,8), 3.57(1 \mathrm{H}, \mathrm{dd}, J 10,4), 3.71(3 \mathrm{H}, \mathrm{s})$, $4.42(1 \mathrm{H}, \mathrm{d}, J 10), 5.97(1 \mathrm{H}, \mathrm{dd}, J 16,1), 6.70(2 \mathrm{H}, \mathrm{dd}, J 8,1)$, $6.90(1 \mathrm{H}$, ddd, $J 8,8,1), 7.00(1 \mathrm{H}$, dd, $J 16,8.5)$ and 7.14-7.36 $(6 \mathrm{H}, \mathrm{m}) ; \delta_{\mathrm{C}}\left(\mathrm{CDCl}_{3}\right) 35.2(\mathrm{q}), 37.1(\mathrm{t}), 44.4(\mathrm{~d}), 51.1(\mathrm{q}), 51.6$ (d), 59.1 (d), 116.4 (s), 117.0 (d, 2 C), 121.2 (d), 121.3 (d), 124.9 (d), 126.3 (d), 127.1 (d), 128.4 (d), 129.3 (d, 2 C), 140.1 (s), 141.3 (s), 149.4 (s), 149.6 (d) and $166.8(\mathrm{~s}) ; \mathrm{m} / z 346\left(28 \%, \mathrm{M}^{+}\right), 320$ (48), $201(40), 169(29)$ and $145(100)$.

27 (2E-configuration): Yellow oil; TLC [EtOAc-hexane $(10: 90)] R_{\mathrm{F}} 0.14 ; v_{\max }($ neat $) / \mathrm{cm}^{-1} 2227(\mathrm{CN}), 1734(\mathrm{CO})$ and $1597 ; \delta_{\mathrm{H}}\left(\mathrm{CDCl}_{3}\right) 2.64-2.88(4 \mathrm{H}, \mathrm{m}), 3.16(3 \mathrm{H}, \mathrm{s}), 3.19-3.33$ $(2 \mathrm{H}, \mathrm{m}), 3.44-3.55(1 \mathrm{H}, \mathrm{m}), 3.59-3.90(1 \mathrm{H}, \mathrm{m}), 3.77(3 \mathrm{H}, \mathrm{s})$, $5.81(1 \mathrm{H}, \mathrm{d}, J 10)$ and $7.02-7.37(9 \mathrm{H}, \mathrm{m}) ; \delta_{\mathrm{C}}\left(\mathrm{CDCl}_{3}\right) 38.0(\mathrm{t})$, 38.6 (t), 40.2 (q), 47.8 (d, 2 C), 51.8 (q), 114.8 (s), 120.7 (d, 2 C), $122.1(\mathrm{~s}), 123.2(\mathrm{~d}), 123.3(\mathrm{~d}), 124.4$ (d), 126.8 (d), 127.9 (d), 129.2 (d, 2 C), $131.5(\mathrm{~d}), 141.4(\mathrm{~s}), 144.0(\mathrm{~s}), 146.3(\mathrm{~s})$ and $172.9(\mathrm{~s}) ; \mathrm{m} / \mathrm{z}$ $346\left(44 \%, \mathrm{M}^{+}\right)$and $272(100)$ (Found: $\mathrm{M}^{+}, 346.1672$. Calc. for $\mathrm{C}_{22} \mathrm{H}_{22} \mathrm{~N}_{2} \mathrm{O}_{2}: M, 346.1681$ ).

trans-3-\{1-[Cyano ( $N$-methylanilino)methyl]-2,3-dihydro-1methylindan-2-yl\}but-2-enenitrile 28

The free-radical cyclisation of 14 [a mixture of $(2 E, 5 E)$ - and $(2 Z, 5 E)$-isomers $(50: 50), 190 \mathrm{mg}]$, by a procedure similar to that for 17, gave a mixture of $\mathbf{2 8 a}$ and $\mathbf{2 8 b}(95 \mathrm{mg}, 55: 45,62 \%$ ). Analytical samples of $\mathbf{2 8 a}$ and $\mathbf{2 8 b}$ were obtained by HPLC. 28a: Yellow oil; TLC [EtOAc-hexane (10:90)] $R_{\mathrm{F}} 0.16 ; v_{\max }$ (neat)/ $\mathrm{cm}^{-1} 2215 ; \delta_{\mathrm{H}}\left(\mathrm{CDCl}_{3}\right) 1.37(3 \mathrm{H}, \mathrm{s}), 1.79(3 \mathrm{H}, \mathrm{d}, J 1), 2.46$ $(3 \mathrm{H}, \mathrm{s}), 2.75(1 \mathrm{H}, \mathrm{dd}, J 18,13), 2.82(1 \mathrm{H}, \mathrm{dd}, J 18,6), 3.26(1 \mathrm{H}$, $\mathrm{dd}, J 13,6), 4.54(1 \mathrm{H}, \mathrm{s}), 5.08(1 \mathrm{H}, \mathrm{d}, J 1), 6.54(2 \mathrm{H}, \mathrm{d}, J 8), 6.82$ $(2 \mathrm{H}, \mathrm{dd}, J 8,1), 7.10-7.24(4 \mathrm{H}, \mathrm{m})$ and $7.35(1 \mathrm{H}, \mathrm{dd}, J 8,1)$; $\delta_{\mathrm{C}}\left(\mathrm{CDCl}_{3}\right) 18.8(\mathrm{q}), 20.4(\mathrm{q}), 35.8(\mathrm{t}), 37.1(\mathrm{q}), 53.6(\mathrm{~d}), 55.8(\mathrm{~s})$, $63.0(\mathrm{~d}), 98.4(\mathrm{~d}), 115.6(\mathrm{~d}, 2 \mathrm{C}), 115.9(\mathrm{~s}), 120.3(\mathrm{~s}), 120.8(\mathrm{~d})$, 124.5 (d), 124.9 (d), 127.7 (d), 129.4 (d, 2 C), 129.6 (d), 142.0 (s), $143.6(\mathrm{~s}), 150.0(\mathrm{~s})$ and $164.8(\mathrm{~s}) ; \mathrm{m} /=341\left(27 \%, \mathrm{M}^{+}\right), 315$ (23), $196(61), 146(55)$ and $145(100) .28 b$ : Yellow oil; TLC [EtOAc-hexane $(10: 90)] R_{\mathrm{F}} 0.15 ; v_{\max }($ neat $) / \mathrm{cm}^{-1} 2214(\mathrm{CN})$; $\delta_{\mathrm{H}}\left(\mathrm{CDCl}_{3}\right) 1.53(3 \mathrm{H}, \mathrm{s}), 1.86(3 \mathrm{H}, \mathrm{d}, J 1, \mathrm{Me}) .2 .78(1 \mathrm{H}, \mathrm{dd}, J$ $14,9), 3.03(3 \mathrm{H}, \mathrm{s}), 3.32(1 \mathrm{H}, \mathrm{dd}, J 14,5), 4.00(1 \mathrm{H}, \mathrm{dd}, J 9,5)$, $4.44(1 \mathrm{H}, \mathrm{s}), 5.16(1 \mathrm{H}, \mathrm{d}, J 1), 6.90(2 \mathrm{H}, \mathrm{dd}, J 8,1), 6.94-7.02(2$ $\mathrm{H}, \mathrm{m})$ and $7.25-7.40(5 \mathrm{H}, \mathrm{m}) ; \delta_{\mathrm{C}}\left(\mathrm{CDCl}_{3}\right) 18.8(\mathrm{q}), 19.4(\mathrm{q}), 35.4$ (t), 38.4 (q), 52.7 (d), 56.8 (s), 62.6 (d), $98.3(\mathrm{~d}), 115.9$ (s), 116.5 (d, 2 C), 120.3 (s), 121.3 (d), 124.7 (d), 124.9 (d, 127.7 (d), 129.0 (d), $129.7(\mathrm{~d}, 2 \mathrm{C}), 141.2(\mathrm{~s}), 144.7(\mathrm{~s}), 150.5(\mathrm{~s})$ and $165.2(\mathrm{~s}) ; \mathrm{m} / \mathrm{z}$ $341\left(5 \%, \mathrm{M}^{+}\right), 315(28), 196(63), 181(22), 146(46)$ and 145 (100) (Found: $\mathrm{M}^{+}$, 341.1891. Calc. for $\mathrm{C}_{23} \mathrm{H}_{23} \mathrm{~N}_{3}: M$, 341.1892).

\section{2-( $N$-Methylanilino)-2-prop-1-enylcyclohexylacetonitrile 29}

The free-radical cyclisation of 15 [a mixture of $(2 E, 5 E)$ - and $(2 Z, 5 E)$-isomers $(38: 62), 174 \mathrm{mg})$, by a procedure similar to that for 17, gave 29a $(14 \mathrm{mg}, 11 \%)$ and $29 \mathrm{~b}(104 \mathrm{mg}, 77 \%)$. 29a: Yellow oil; TLC [EtOAc-hexane (2:98)] $R_{\mathrm{F}} 0.09$; $v_{\max }($ neat $) / \mathrm{cm}^{-1} 2224 ; \delta_{\mathrm{H}}\left(\mathrm{CDCl}_{3}\right) 1.02-1.35(2 \mathrm{H}, \mathrm{m}), 1.42-$ $1.82(5 \mathrm{H}, \mathrm{m}), 1.73(3 \mathrm{H}, \mathrm{d}, J 5.5), 1.90-2.05(1 \mathrm{H}, \mathrm{m}), 2.62-2.72$ ( $\mathrm{l} \mathrm{H}, \mathrm{m}), 2.85(3 \mathrm{H}, \mathrm{s}), 3.00-3.12(1 \mathrm{H}, \mathrm{m}), 4.14(1 \mathrm{H}, \mathrm{d}, J 8,2-\mathrm{H})$, $5.61-5.72(2 \mathrm{H}, \mathrm{m}), 6.85(2 \mathrm{H}, \mathrm{d}, J 8), 6.88(1 \mathrm{H}, \mathrm{dd}, J 8,8)$ and $7.26(2 \mathrm{H}, \mathrm{dd}, J 8,8) ; \delta_{\mathrm{C}}\left(\mathrm{CDCl}_{3}\right) 18.3(\mathrm{q}), 20.9(\mathrm{t}), 23.9(\mathrm{t}), 25.9$ 
$(\mathrm{t}), 32.5(\mathrm{t}), 34.6(\mathrm{q}), 39.4(\mathrm{~d}), 41.6(\mathrm{~d}), 58.5(\mathrm{~d}, \mathrm{C}-2), 116.2(\mathrm{~d}, 2 \mathrm{C})$, 117.1 (s), 120.5 (d), 121.7 (d), 129.1 (d), 129.3 (d, 2 C) and 149.6 (s); $m / z 268\left(3 \%, \mathrm{M}^{+}\right), 146(30)$ and 145 (100). 29b: White solid, mp $46-47^{\circ} \mathrm{C}$; TLC [EtOAc-hexane $(2: 98)$ ] $R_{\mathrm{F}} 0.07$; $v_{\max }(\mathrm{KBr}) / \mathrm{cm}^{-1} 2223 ; \delta_{\mathrm{H}}\left(\mathrm{CDCl}_{3}\right) 1.11-1.40(4 \mathrm{H}, \mathrm{m}), 1.64$ $(3 \mathrm{H}, \mathrm{d}, J 5), 1.59-2.04(6 \mathrm{H}, \mathrm{m}), 2.85(3 \mathrm{H}, \mathrm{s}), 4.29(1 \mathrm{H}, \mathrm{d}, J 7.5$, 2-H), 5.35-5.53 (2 H, m), 6.86-6.91 (3 H, m) and 7.27 (2 H, dd, $J$ $8,8) ; \delta_{\mathrm{C}}\left(\mathrm{CDCl}_{3}\right) 17.7(\mathrm{q}), 24.9(\mathrm{t}), 25.1(\mathrm{t}), 27.4(\mathrm{t}), 33.1(\mathrm{t}), 35.1$ (q), 44.0 (d), 45.8(d), 57.6 (d, C-2), 116.2 (d, 2 C), 117.9 (s), 120.2 (d), 126.3 (d), $129.2(\mathrm{~d}, 2 \mathrm{C}), 134.7$ (d) and $149.8(\mathrm{~s}) ; \mathrm{m} / \mathrm{z} 268(8 \%$, $\mathrm{M}^{+}$), 146 (32) and 145 (100) (Found: $\mathrm{M}^{+}, 268.1935$. Calc. for $\mathrm{C}_{18} \mathrm{H}_{24} \mathrm{~N}_{2}: M, 268.1939$ ).

\section{2-(2-Methylcyclohex-3-enyl)-2-( $N$-methylanilino)acetonitrile \\ 30}

The free-radical cyclisation of 16 [a mixture of $(2 E, 5 E)$ - and $(2 Z, 5 E)$-isomers $(43: 57), 70 \mathrm{mg}$ ], by a procedure similar to that for 17 , gave $30 \mathrm{a}(12 \mathrm{mg}, 22 \%), 30 \mathrm{~b}(5 \mathrm{mg}, 10 \%)$ and $30 \mathrm{c}(4 \mathrm{mg}$, $8 \%)$. The starting material 16 was recovered $(30 \%, E / Z=2: 1)$. 30a: Yellow oil; TLC [EtOAc-hexane (2:98)] $R_{\mathrm{F}} \quad 0.09$; $v_{\max }($ neat $) / \mathrm{cm}^{-1} 2212 ; \delta_{\mathrm{H}}\left(\mathrm{CDCl}_{3}\right) 1.03(3 \mathrm{H}, \mathrm{d}, J 7), 1.29$ $1.47(2 \mathrm{H}, \mathrm{m}), 1.80-1.93(1 \mathrm{H}, \mathrm{m}), 1.98-2.12(2 \mathrm{H}, \mathrm{m}), 2.17-2.36$ $(1 \mathrm{H}, \mathrm{m}), 2.46-2.65(1 \mathrm{H}, \mathrm{m}), 2.89(3 \mathrm{H}, \mathrm{s}), 4.26(1 \mathrm{H}, \mathrm{d}, J 11.5$, 2-H), 5.68-5.74 (2 H, m), 6.80-6.97 (3 H, m) and 7.24-7.34 (2 H, $\mathrm{m}) ; \delta_{\mathrm{C}}\left(\mathrm{CDCl}_{3}\right) 18.0(\mathrm{q}), 19.3(\mathrm{t}), 25.5(\mathrm{t}), 35.5(\mathrm{~d}), 34.3(\mathrm{q}), 38.7$ (d), 57.3 (d, C-2), 116.6 (d, 2 C), 116.9 (s), 120.6 (d), 126.1 (d), $129.3(\mathrm{~d}, 2 \mathrm{C}), 132.2(\mathrm{~d})$ and $150.1(\mathrm{~s}) ; \mathrm{m} / z 240\left(15 \%, \mathrm{M}^{+}\right)$and 145 (100) (Found: $\mathrm{M}^{+}, 240.1623$. Calc. for $\mathrm{C}_{16} \mathrm{H}_{20} \mathrm{~N}_{2}: M$, 240.1626). 30b: Yellow oil; TLC [EtOAc-hexane (2:98)] $R_{\mathrm{F}}$ $0.07 ; v_{\max }($ neat $) / \mathrm{cm}^{-1} 2224 ; \delta_{\mathrm{H}}\left(\mathrm{CDCl}_{3}\right) 1.11(3 \mathrm{H}, \mathrm{d}, J 7)$, 1.57-1.76 (1 H, m), 1.86-2.20 (4 H, m), 2.30-2.50 (1 H, m), 2.88 ( $3 \mathrm{H}, \mathrm{s}), 4.52(1 \mathrm{H}, \mathrm{d}, J 11,2-\mathrm{H}), 5.52-5.79(2 \mathrm{H}, \mathrm{m}), 6.85-6.94$ $(3 \mathrm{H}, \mathrm{m})$ and $7.23-7.33(2 \mathrm{H}, \mathrm{m}) ; \delta_{\mathrm{C}}\left(\mathrm{CDCl}_{3}\right) 20.0(\mathrm{t}), 21.1(\mathrm{t})$, 21.3 (q), 29.2 (d), 34.2 (q), 39.2 (d), 56.5 (d, C-2), 116.2 (d, 2 C), 117.1 (s), 120.5 (d), 125.7 (d), 129.3 (d, 2 C), 130.7 (d) and 149.8 (s); $m / z 240\left(6 \%, \mathrm{M}^{+}\right)$and $145(100)$. 30c: Yellow oil; TLC [EtOAc-hexane $(2: 98)] \quad R_{\mathrm{F}} \quad 0.06 ; \quad v_{\max }$ (neat) $/ \mathrm{cm}^{-1} \quad 2225$; $\delta_{\mathrm{H}}\left(\mathrm{CDCl}_{3}\right) 1.18(3 \mathrm{H}, \mathrm{d}, J 7), 1.69-1.77(2 \mathrm{H}, \mathrm{m}), 1.83-1.91$ (2 H, m), 2.03-2.19 (1 H, m), 2.25-2.40 (1 H, m), $2.88(3 \mathrm{H}, \mathrm{s})$, $4.44(1 \mathrm{H}, \mathrm{d}, J 11,2-\mathrm{H}), 5.56-5.75(2 \mathrm{H}, \mathrm{m}), 6.88-6.94(3 \mathrm{H}, \mathrm{m})$ and 7.24-7.33 (2 H, m); $\delta_{\mathrm{C}}\left(\mathrm{CDCl}_{3}\right) 18.1(\mathrm{q}), 20.8(\mathrm{t}), 21.6(\mathrm{t})$, $31.3(\mathrm{~d}), 34.1$ (q), 39.9 (d), 55.4 (d, C-2), $116.2(\mathrm{~d}, 2 \mathrm{C}), 116.5(\mathrm{~s})$,
120.6 (d), 125.8 (d), 129.4 (d, 2 C), 130.3 (d) and 149.2 (s); $m / z$ $240\left(6 \%, \mathrm{M}^{+}\right)$and $145(100)$.

\section{Acknowledgements}

We thank the National Science Council for financial support (NSC 83-0208-M002-041) and Prof. Yu Wang (National Taiwan University) for the X-ray diffraction analyses.

\section{References}

1 (a) J.-M. Fang, H.-T. Chang and C.-C. Lin, J. Chem. Soc., Chem. Commun., 1988, 1385; (b) C.-C. Yang, H.-T. Chang and J.-M. Fang, J. Org. Chem., 1993, 58, 3100.

2 (a) A. L. J. Beckwith, J. Chem. Soc., Chem. Commun., 1980, 482; (b) A. L. J. Beckwith and C. H. Schiesser, Tetrahedron, 1985, 41, 3925.

3 (a) S.-U. Park, S.-K. Chung and M. Newcomb, J. Am. Chem. Soc., $1986,108,240 ;(b)$ M. Newcomb, T. R. Varick, C. Ha, M. B. Manek and X. Yue, J. Am. Chem. Soc., 1992, 114, 8158; (c) B. Giese, Angew. Chem., Int. Ed. Engl., 1983, 22, 753; (d) M. W. Wong, A. Pross and L. Radom, J. Am. Chem. Soc., 1993, 115, 11050.

4 (a) F. G. Bordwell, M. J. Bausch, J. P. Cheng, T. H. Crip, T. Y. Lynch and M. E. Mueller, J. Org. Chem., 1990, 55, 58; (b) K. Pius and J. Chandrasekhar, J. Chem. Soc., Chem. Commun., 1990, 41.

5 (a) A. T. Balaban, R. Baican and N. Neguita, Tetrahedron Lett., 1973, 1877; (b) A. T. Balaban and R. Istratiu, Tetrahedron Lett., 1973,$1879 ;(c)$ D. Crans, T. Clark and P. V. R. Schleyer, Tetrahedron Lett., 1980, 21, 3681; (d) D. J. Pasto, J. Am. Chem. Soc., 1988, 110, 8164; (e) H. G. Viehe, Z. Janousek, R. Merenyi and L. Stella, Acc. Chem. Res., 1985, 18, 148; $(f)$ H. G. Viehe, R. Merenyi and Z. Janousek, Pure Appl. Chem., 1988, 60, 1635.

6 J.-M. Fang, C.-C. Yang and Y.-W. Wang, J. Org. Chem., 1989, 54, 477.

7 J.-M. Fang and C.-C. Yang, J. Chem. Soc., Perkin Trans. 1, 1992, 3085.

8 (a) J. D. Albright, Tetrahedron, 1983, 39, 3207; (b) W. H. Bunnelle and C. G. Shevlin, Tetrahedron Lett., 1989, 30, 4203 and references cited therein; (c) S. Arseniyadis, K. S. Kyler and D. S. Watt, Org. Reactions, 1984, 31, 1; (d) T.-S. Chuang, C.-C. Yang, C.-J. Chang and J.-M. Fang, Synlett, 1990, 733.

9 A. N. Kurtz, W. E. Billups, R. B. Greenlee, H. F. Hamil and W. T. Pace, J. Org. Chem., 1965, 30, 3141.

Paper 4/05916F

Received 28th September 1994 Accepted 5th December 1994 